\title{
Quantum Entanglement in Neural Network States
}

\author{
Dong-Ling Deng, ${ }^{1, *}$ Xiaopeng Li, ${ }^{2,3,1}$ and S. Das Sarma ${ }^{1}$ \\ ${ }^{1}$ Condensed Matter Theory Center and Joint Quantum Institute, Department of Physics, \\ University of Maryland, College Park, Maryland 20742-4111, USA \\ ${ }^{2}$ State Key Laboratory of Surface Physics, Institute of Nanoelectronics and Quantum Computing, \\ and Department of Physics, Fudan University, Shanghai 200433, China \\ ${ }^{3}$ Collaborative Innovation Center of Advanced Microstructures, Nanjing 210093, China \\ (Received 25 January 2017; revised manuscript received 18 March 2017; published 11 May 2017)
}

\begin{abstract}
Machine learning, one of today's most rapidly growing interdisciplinary fields, promises an unprecedented perspective for solving intricate quantum many-body problems. Understanding the physical aspects of the representative artificial neural-network states has recently become highly desirable in the applications of machine-learning techniques to quantum many-body physics. In this paper, we explore the data structures that encode the physical features in the network states by studying the quantum entanglement properties, with a focus on the restricted-Boltzmann-machine (RBM) architecture. We prove that the entanglement entropy of all short-range RBM states satisfies an area law for arbitrary dimensions and bipartition geometry. For long-range RBM states, we show by using an exact construction that such states could exhibit volume-law entanglement, implying a notable capability of RBM in representing quantum states with massive entanglement. Strikingly, the neural-network representation for these states is remarkably efficient, in the sense that the number of nonzero parameters scales only linearly with the system size. We further examine the entanglement properties of generic RBM states by randomly sampling the weight parameters of the RBM. We find that their averaged entanglement entropy obeys volume-law scaling, and the meantime strongly deviates from the Page entropy of the completely random pure states. We show that their entanglement spectrum has no universal part associated with random matrix theory and bears a Poisson-type level statistics. Using reinforcement learning, we demonstrate that RBM is capable of finding the ground state (with power-law entanglement) of a model Hamiltonian with a longrange interaction. In addition, we show, through a concrete example of the one-dimensional symmetryprotected topological cluster states, that the RBM representation may also be used as a tool to analytically compute the entanglement spectrum. Our results uncover the unparalleled power of artificial neural networks in representing quantum many-body states regardless of how much entanglement they possess, which paves a novel way to bridge computer-science-based machine-learning techniques to outstanding quantum condensed-matter physics problems.
\end{abstract}

DOI: 10.1103/PhysRevX.7.021021

Subject Areas: Computational Physics,

Condensed Matter Physics, Quantum Physics

\section{INTRODUCTION}

Understanding the behavior of quantum many-body systems beyond the standard mean-field paradigm is a central (and daunting) task in condensed-matter physics. One challenge lies in the exponential scaling of the Hilbert space dimension [1-3]. In principle, a complete description of a generic many-body state requires an exponential amount of information, rendering the problem unattainable,

\footnotetext{
* Corresponding author. dldeng15@umd.edu

Published by the American Physical Society under the terms of the Creative Commons Attribution 4.0 International license. Further distribution of this work must maintain attribution to the author(s) and the published article's title, journal citation, and DOI.
}

even numerically. Fortunately, physical states usually only access a tiny corner of the entire Hilbert space and can often be characterized with much less classical resources. Constructing efficient representations of such states is thus of crucial importance in tackling quantum many-body problems. Notable examples include quantum states with area-law entanglement [4], such as ground states of local gapped Hamiltonians [5] or the eigenstates of many-body localized systems [6], which can efficiently be represented in terms of matrix product states (MPS) [7-9] or tensornetwork states, in general [10-12]. These compact representations of quantum states play a vital role and are indispensable for tackling a variety of many-body problems ranging from the classification of topological phases $[13,14]$ to the construction of the AdS/CFT correspondence $[15,16]$. In addition, they are also the backbones of a number of efficient classical algorithms for solving intricate 
many-body problems, e.g., density-matrix renormalization group (DMRG) $[9,17,18]$, time-evolving block decimation (TEBD) [19], projected entangled pair states (PEPS) $[10,12]$, and multiscale entanglement renormalization ansatz (MERA) methods [20,21]. Recently, a novel neural-network representation of quantum many-body states has been introduced [22] in solving many-body problems with machine-learning techniques. However, the entanglement properties (which are crucial for the renowned MPS or tensor-network representations) of these neural-network states remain unknown. In this paper, we fill this crucial gap by studying the entanglement properties of these many-body neural-network quantum states both analytically and numerically. Our work provides an important connection between the physical properties of manybody quantum entanglement and the computer-science properties of neural-network-based machine leaning.

Machine learning is the core of artificial intelligence and data science [23]. It powers many aspects of modern society, and its applications have become ubiquitous throughout science, technology, and commerce [24,25]. In fact, perhaps because of the dominant presence of big data in our modern world, the terms artificial intelligence, machine learning, neural networks, deep learning, etc. have generically entered the lexicon of the cultural world, well outside the technical world of computer science where they originated, often appearing in everyday press and popular articles or stories-for example, the software technology underlying automated self-driving cars crucially depends on artificial intelligence and machine learning. Within physics, applications of machine-learning techniques have recently been invoked in various contexts such as gravitational wave analysis [26,27], black hole detection [28], material design [29], and classification of the classical liquid-gas transitions [30]. Very recently, these techniques have been introduced to many-body quantum condensedmatter physics, raising considerable interest across different communities [22,31-44]. Exciting progress has been made in identifying quantum phases and transitions among them (either conventional symmetry-broken $[33,35,37,38]$ or topological phases [32]), modeling thermodynamic observables [36], constructing decoders for topological codes [39], accelerating Monte Carlo simulations [41,42], and establishing connections to renormalization group techniques $[45,46]$, etc. In addition, machine-learning ideas have also been explored in measuring quantum entanglement and wave-function tomography through the analyses of data extracted from quantum gas microscopes in coldatom experiments [47]. The fledgling field of machinelearning applications in physics appears to be in its rapidly growing early phase with many future breakthroughs expected as it matures.

From the numerical perspective, the applications of machine-learning techniques to many-body problems would rely vitally on the underlying data structures of the artificial neural networks, whose connections to the entanglement features of the corresponding quantum states are particularly desirable to address. In this paper, we study the entanglement properties, such as the entanglement entropy and spectrum, of the neural-network states. We focus on the quantum states represented by the restricted Boltzmann machine (RBM), which is a stochastic artificial neural network with widespread applications [40,48-50]. We first prove the general result that all short-range RBM states obey an entanglement area law, independent of dimensionality and bipartition geometry. Since the onedimensional (1D) symmetry-protected topological (SPT) cluster states and toric code states (in both 2D and 3D) have an exact short-range RBM representation [51], it follows immediately that they all have area-law entanglement. For long-range RBM states, calculating their entanglement entropy and spectrum analytically is very challenging (if not impossible), and we thus resort to numerical simulations. We randomly sample the weight parameters of the RBM states and compute their entanglement entropy and spectrum. We find that their entanglement entropy exhibits a volume-law scaling, in general. However, surprisingly, their entropy is noticeably less than the Page entropy for random pure states, and their entanglement spectrum has no universal part associated with random matrix theory and bears a Poisson-type level statistics. This indicates that the RBM states with random weight parameters live in a very restricted subspace of the entire Hilbert space (in spite of manifesting a volume-law entanglement entropy) and are not irreversible-namely, there exists an efficient algorithm to completely disentangle these states [52].

In addition, we analytically construct a family of RBM states with maximal volume-law entanglement. These states cannot be described in terms of matrix product states or tensor-network states with a computationally tractable bond dimension. In sharp contrast, their RBM representation is remarkably efficient, requiring only a small number of parameters that scales linearly with the system size. This shows, in an exact fashion and in the most explicit way, the unparalleled power of neural networks in describing many-body quantum states with large entanglement. Unlike MPS or tensor-network states, entanglement is not the limiting factor for the efficiency of the neuralnetwork representation. As an important consequence, we are able to calculate (through a reinforcement-learning scheme [22,53]) the ground state, whose entanglement has a power-law scaling with system size, of a spin Hamiltonian with long-range interaction. Finally, we show that the RBM representation could also be used as a tool to analytically compute the entanglement entropy and spectrum for certain quantum states with short-range RBM descriptions. We demonstrate this by using a concrete example of the 1D SPT cluster states. Our results not only demonstrate explicitly the exceptional power of artificial neural networks in representing quantum many-body states, but they also 
reveal some crucial aspects of their data structures, which provide a valuable guide for the emerging new field of machine learning and many-body quantum physics.

\section{NEURAL-NETWORK REPRESENTATION AND QUANTUM ENTANGLEMENT: CONCEPTS AND NOTATIONS}

An artificial-neural-network representation of quantum many-body states has recently been introduced by Carleo and Troyer in Ref. [22], where they demonstrated the remarkable power of a reinforcement-learning approach in calculating the ground state or simulating the unitary time evolution of complex quantum systems with strong interactions. We show elsewhere that this representation can be used to describe topological states, even for those with long-range entanglement [51]. To start with, let us first briefly introduce this representation in the RBM architecture. We consider a quantum system with $N$ spins living on a $d$-dimensional cubic lattice $\Xi=\left(\sigma_{\mathbf{r}_{1}}, \sigma_{\mathbf{r}_{2}}, \ldots, \sigma_{\mathbf{r}_{N}}\right)$. Correspondingly, we introduce $\Xi_{Y}$ for spins in a subsystem $Y$ as $\Xi_{Y}=\left\{\sigma_{\mathbf{r}}: \mathbf{r} \in Y\right\}$. The geometric details of the lattice do not matter. Here, we choose cubic lattices and focus on spin- $\frac{1}{2}$ (qubits) systems for simplicity. A RBM neural network contains two layers, one visible layer with $N$ nodes (visible neurons) corresponding to the physical spins, the other a hidden layer with $M$ auxiliary nodes $\left(h_{\mathbf{r}_{1}}, h_{\mathbf{r}_{2}}, \ldots, h_{\mathbf{r}_{M}}\right)$ (hidden neurons). The neurons in the hidden layer are connected to those in the visible layer, but there is no connection among neurons in the same layer (see Fig. 1 for a 2D illustration). The RBM neural-network representation of a quantum state is obtained by tracing out the hidden neurons [22]:

$$
\Phi_{M}(\Xi ; \Omega)=\sum_{\left\{h_{\mathbf{r}}\right\}} e^{\sum_{\mathbf{r}} a_{\mathbf{r}} \sigma_{\mathbf{r}}^{2}+\sum_{\mathbf{r}^{\prime}} b_{\mathbf{r}^{\prime}} h_{\mathbf{r}^{\prime}}+\sum_{\mathbf{r r}^{\prime}} W_{\mathbf{r}^{\prime} \mathbf{r}} h_{\mathbf{r}^{\prime}} \sigma_{\mathbf{r}}^{2}}
$$

where $\left\{h_{\mathbf{r}}\right\}=\{-1,1\}^{M}$ denotes the possible configurations of the hidden spin variables and the weights $\Omega=$ $\left(a_{\mathbf{r}}, b_{\mathbf{r}^{\prime}}, W_{\mathbf{r r}^{\prime}}\right)$ are parameters that need to be trained to best represent the many-body quantum state. It is worthwhile to mention that the RBM state defined in Eq. (1) is a variational state, with its amplitude and phase specified by $\Phi_{M}(\Xi ; \Omega)$. The actual quantum state should be understood as (up to an irrelevant normalization constant) $|\Psi(\Omega)\rangle \equiv \sum_{\Xi} \Phi_{M}(\Xi ; \Omega)|\Xi\rangle$, similar to the Laughlin-like description of the resonating-valence-bond ground state of the exactly solvable Haldane-Shastry model [54,55].

We remark that RBMs can be trained in either supervised or unsupervised ways, and in the machine-learning community, RBMs have had successful applications in classification [50], dimensionality reduction [48], feature learning [56], and collaborative filtering [49], etc. Mathematically, the ability of the RBM to approximate any many-body state is assured by representability

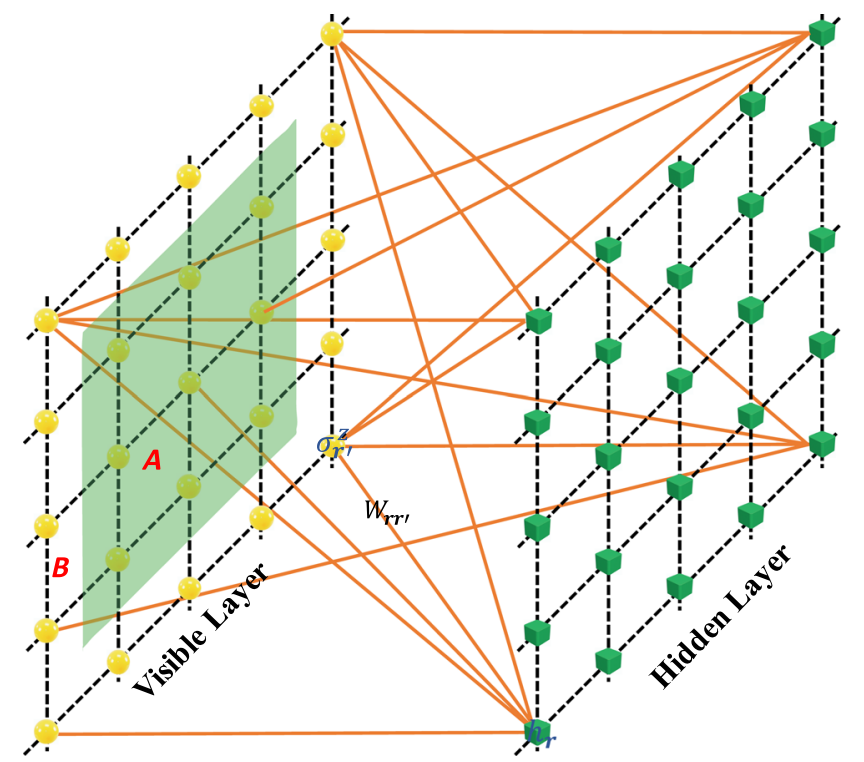

FIG. 1. A 2D pictorial illustration of artificial-neural-network quantum states in the restricted-Boltzmann-machine architecture. The yellow balls (green cubes) denote the neurons on the visible (hidden) layer, corresponding to the physical (auxiliary) spins. The brown lines show the connections between visible and hidden neurons, with the weight parameter denoted by $W_{\mathbf{r r}^{\prime}}$ (only a small portion of the connections are shown for the best visualization). Here, we also show a typical bipartition of the system into two subsystems $A$ and $B$ in order to study the entanglement properties of the neural-network states.

theorems [57-59]. Nevertheless, the approximation may require a huge number (exponential in system size) of neurons and parameters, thus rendering the representation impractical, especially in numerical simulations. A question of both theoretical and practical interest is as follows: What kind of many-body quantum states can be efficiently described by RBMs with a numerically feasible number of neurons and parameters? It is now established that entanglement plays a crucial role in determining whether a quantum state can be efficiently represented by a MPS or tensor network or not. Quantum states with volume-law entanglement cannot be described efficiently by a MPS or tensor network and thus cannot be simulated efficiently by DMRG, PEPS, or MERA. In sharp contrast, as we will show in the following sections, RBMs are indeed capable of efficiently describing certain specific quantum states with volume-law entanglement, giving rise to the great potential of numerically simulating these states with new machine-learning algorithms based on RBMs.

In this paper, we study the quantum entanglement properties of the RBM states. In particular, we investigate the entanglement entropy, the Rényi entropy [60], and the entanglement spectrum [61], which are three of the most broadly used quantities for characterizing many-body entanglement of a pure quantum state. These quantities can be defined as follows: Considering a pure many-body 
quantum state $|\psi\rangle$, we divide the system into two subregions, $A$ and $B$ (a typical bipartition of a $2 \mathrm{D}$ system is shown in Fig. 1). We then construct the reduced density matrix of subsystem $A$ by tracing out the degree of freedom in $B: \rho_{A}(|\psi\rangle)=\operatorname{Tr}_{B}(|\psi\rangle\langle\psi|)$. The $\alpha$ th order Rényi entropy is defined as

$$
S_{\alpha}^{A} \equiv \frac{1}{1-\alpha} \log \left[\operatorname{Tr}\left(\rho_{A}^{\alpha}\right)\right] .
$$

The zeroth-order ( $\alpha=0$ ) Rényi entropy is related to the rank, namely, the number of nonzero singular values of $\rho_{A}$. When $\alpha \rightarrow 1$, the first-order Rényi entropy reduces to the von Neumann entropy,

$$
S_{1}^{A} \equiv-\operatorname{Tr}\left(\rho_{A} \log \rho_{A}\right) .
$$

In the literature, the entanglement entropy usually means the von Neumann entropy. However, throughout this paper, we do not differentiate between the entanglement entropy and the Rényi entropy since most of the results are valid for the Rényi entropy to all orders. To define the entanglement spectrum, we first define the entanglement Hamiltonian by taking the $\log$ of $\rho_{A}$ :

$$
H_{\mathrm{ent}} \equiv-\log \rho_{A},
$$

and then the entanglement spectrum is defined as the spectrum of $H_{\text {ent }}$. We mention that entanglement is nowadays a central concept in many branches of quantum physics. In condensed-matter physics, the entanglement entropy and spectrum have proven to be powerful tools characterizing topological phases [4,61-64], quantum phase transitions [65-67], and many-body localization [68-70], etc. A number of theoretical proposals have been introduced to measure the entanglement entropy [71-74] and spectrum [75] in many-body systems. Notably, experimental measurements of the second-order Rényi entropy have been achieved in recent cold-atom experiments in optical lattices [76,77]. We expect that our study of entanglement properties of neural-network states would also provide novel inspiration in this context.

\section{AREA-LAW ENTANGLEMENT FOR SHORT-RANGE NEURAL-NETWORK STATES}

We start with short-range RBM states and prove that they obey an area-law entanglement scaling; namely, the amount of entanglement between a subsystem and its complement scales, at most, as the surface area or the boundary rather than the volume of the subsystem [4]. Historically, the study of entanglement area laws is inspired by the holographic principle in black hole physics, where the Bekenstein-Hawking entropy of a black hole is believed to scale as its boundary surface rather than its volume [78]. It has been argued that the origin of the black hole entropy is the quantum entanglement between the inside and the outside of the black hole [79-81]. Although it is apparent that entanglement in "natural" quantum systems should roughly live on the boundary and many numerical simulations indeed support this intuition, rigorously proving the area law for a given family of quantum states is notoriously challenging and often involves sophisticated mathematical techniques [4]. A breakthrough was first made by Hastings in Ref. [5], where he proved an entanglement area law for the ground states of 1D gapped local Hamiltonians by using the Lieb-Robinson bound [82]. More recently, this proof has been simplified and generalized to ground states with a finite number of degeneracy by a combinatorial approach based on Chebyshev polynomials $[83,84]$. Unfortunately, both the Lieb-Robinson bound approach and the combinatorial approach seem unlikely to carry over to the case of higher dimensions. Establishing the area-law entanglement for ground states of gapped Hamiltonians in more than one dimension remains a major open problem (and arguably the most important one) in the field of Hamiltonian complexity [3].

Here, we prove that short-range RBM states obey the area law of entanglement in any dimension for arbitrary bipartition geometry. To be precise, we call a RBM state an $\mathcal{R}$-range RBM state if each hidden neuron is only connected to these visible neurons within an $\mathcal{R}$ neighborhood; i.e., $W_{\mathbf{r r}^{\prime}}=0$ if $\left|\mathbf{r}-\mathbf{r}^{\prime}\right|>\mathcal{R}$. For instance, in Ref. [51], we have demonstrated that both the 1D SPT cluster states and toric code states (both 2D and 3D) can be represented exactly by RBMs, with hidden neurons being connected only to nearest visible neurons. These states are 1-range RBM states. For general $\mathcal{R}$-range RBM states, we have the following theorem:

Theorem 1.-For an $\mathcal{R}$-range RBM state, the Rényi entropy for all orders satisfies

$$
S_{\alpha}^{A} \leq 2 \mathcal{S}(A) \mathcal{R} \log 2, \quad \forall \alpha,
$$

where $\mathcal{S}(A)$ denotes the surface area of subsystem $A$. This area law is valid in any dimension and for arbitrary bipartition geometry.

Proof.-For RBMs, since there is no intralayer connections between neurons, we can explicitly factor out the hidden variables and rewrite $\Phi_{M}(\Xi ; \Omega)$ in a product form: $\Phi_{M}(\Xi ; \Omega)=\prod_{\mathbf{r}} e^{a_{\mathbf{r}} \sigma_{\mathbf{r}}^{z}} \prod_{\mathbf{r}^{\prime}} \Gamma_{\mathbf{r}^{\prime}}\left(\Xi_{\underline{r}^{\prime}}\right)$, where we have introduced a local subregion notation $\underline{\mathbf{r}_{0}} \equiv\left\{\mathbf{r}:\left|\mathbf{r}-\mathbf{r}_{0}\right|<R\right\}$, and a local function $\Gamma_{\mathbf{r}^{\prime}}\left(\Xi_{\mathbf{r}^{\prime}}\right)=2 \overline{\cosh }\left(b_{\mathbf{r}^{\prime}}+\sum_{\mathbf{r}} W_{\mathbf{r}^{\prime} \mathbf{r}} \sigma_{\mathbf{r}}^{z}\right)$. We call $\Gamma_{\mathbf{r}^{\prime}}\left(\Xi_{\mathbf{r}^{\prime}}\right)$ the $\Gamma_{\mathbf{r}^{\prime}}$ factor for the hidden neuron at $\mathbf{r}^{\prime}$. Moreover, by the definition of an $\mathcal{R}$-range RBM, the values of $\Gamma_{\mathbf{r}^{\prime}}$ factors only depend on the configuration of these visible neurons (physical spins) within an $\mathcal{R}$ neighborhood [denoted by $\mathcal{N}_{\mathbf{r}^{\prime}}(\mathcal{R})$ ]. We can thus simplify $\Gamma_{\mathbf{r}^{\prime}}\left(\Xi_{\mathbf{r}^{\prime}}\right)$ as $\Gamma_{\mathbf{r}^{\prime}}\left(\Xi_{\mathbf{r}^{\prime}}\right)=2 \cosh \left(b_{\mathbf{r}^{\prime}}+\sum_{\mathbf{r} \in \mathcal{N}_{\mathbf{r}^{\prime}}(\mathcal{R})} W_{\mathbf{r}^{\prime} \mathbf{r}} \sigma_{\mathbf{r}}^{z}\right)$. This indicates the locality feature of $\Gamma_{\mathbf{r}^{\prime}}$ factors, which is the origin of the area law. 
In order to utilize the locality feature of $\Gamma_{\mathbf{r}^{\prime}}$ factors, we can further divide the subregion $A(B)$ into three parts: $A_{1}$, $A_{2}$, and $A_{3}\left(B_{1}, B_{2}\right.$, and $\left.B_{3}\right)$, as illustrated in Fig. 2. Explicitly, the subregion with $\mathbf{r}$ directly coupled (via one hidden neuron) to the lattice sites in $B$ is defined to be $A_{3}$, the one directly coupled to $A_{3}$ within $A$ is defined to be $A_{2}$, and the rest of $A$ is $A_{1}$. The subregions $B_{1,2,3}$ are introduced correspondingly. One can regard the subregions $A_{2}, A_{3}, B_{2}$, and $B_{3}$ as hypersurfaces with thickness $\mathcal{R}$ in high dimensions. Let $Y=A_{2} \cup A_{3} \cup B_{2} \cup B_{3}$; then, we can rewrite the $\mathcal{R}$-range $\mathrm{RBM}$ state as

$$
|\Psi(\Omega)\rangle=\sum_{\Xi_{Y}} \prod_{\mathbf{r}^{\prime} \in A_{3} \cup B_{3}} \Gamma_{\mathbf{r}^{\prime}}\left(\Xi_{\mathbf{r}^{\prime}}\right)\left|\varphi_{A}\right\rangle\left|\varphi_{B}\right\rangle,
$$

where $\left|\varphi_{A}\right\rangle=\sum_{\Xi_{A_{1}}} \prod_{\mathbf{r}^{\prime} \in A_{1} \cup A_{2}} \Gamma_{\mathbf{r}^{\prime}}\left(\Xi_{\mathbf{r}^{\prime}}\right)\left|\Xi_{A}\right\rangle$ and $\left|\varphi_{B}\right\rangle=$ $\sum_{\Xi_{B_{1}}} \prod_{\mathbf{r}^{\prime} \in B_{1} \cup B_{2}} \Gamma_{\mathbf{r}^{\prime}}\left(\Xi_{\underline{r}^{\prime}}\right)\left|\Xi_{B}\right\rangle$. From Eq. (3), we only have, at most, $2^{|Y|}$ terms, with $|Y|$ denoting the number of spins in region $Y$, in the summation, and each term is a tensor product of orthogonal states of $A$ and $B$. This gives the upper bound in Eq. (2) after tracing out the degrees of freedom in region $B$. We stress two crucial aspects of Eq. (3): (i) $\left|\varphi_{A}\right\rangle$ is independent of spin configurations in region $B$, and $\left|\varphi_{B}\right\rangle$ is independent of spin configurations in region $A$; (ii) the coefficients $\prod_{\mathbf{r}^{\prime} \in A_{3} \cup B_{3}} \Gamma_{\mathbf{r}^{\prime}}\left(\Xi_{\mathbf{r}^{\prime}}\right)$ for each

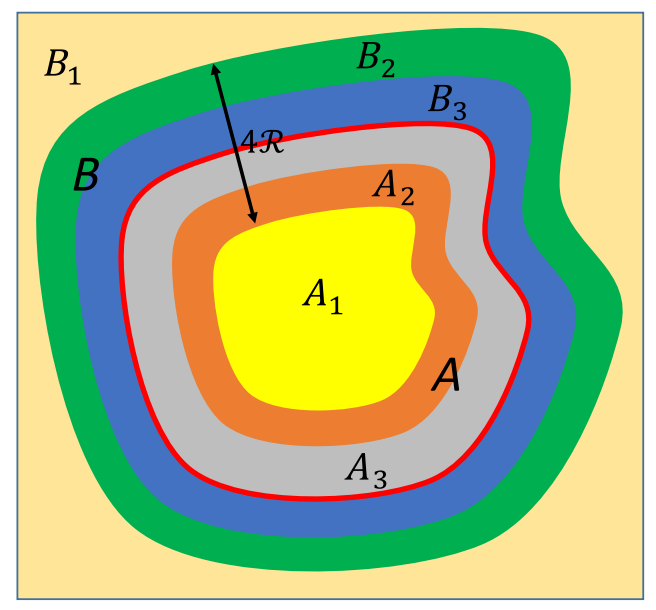

FIG. 2. A sketch for the proof of area-law entanglement for short-range restricted-Boltzmann-machine states. The system is divided into two subsystems, $A$ and $B$, with the red line showing the interface boundary. In order to show that the Rényi entropy $S_{\alpha}^{A}$ obeys an area law for any $\alpha$, the subsystem $A(B)$ is further divided into three parts: $A_{1}, A_{2}$, and $A_{3}\left(B_{1}, B_{2}\right.$, and $\left.B_{3}\right)$. Since the neural network is short range, the $\Gamma_{\mathbf{r}}$ factors with $\mathbf{r} \in A_{1} \cup A_{2}$ ( $\mathbf{r} \in B_{1} \cup B_{2}$ ) are independent of spin configurations in region $B$ $(A)$; thus, we can group the spins in regions $A_{1}$ and $B_{1}$ with their $\Gamma_{\mathbf{r}}$ factors. The entropy of the reduced density matrix $\rho_{A}$ then only depends on the degree of freedom in region $A_{2} \cup A_{3}$, which is proportional to the surface area of region $A$. This gives us a clear geometric picture of why $S_{\alpha}^{A}$ is upper bounded by the surface area of $A$, up to an unimportant scaling constant as given in Eq. (2). orthogonal component $\left|\varphi_{A}\right\rangle\left|\varphi_{B}\right\rangle$ are independent of spin configurations outside $Y$. Points (i) and (ii) are crucial for the validity of the proof, and they are made evident by the deliberate partitions of both subregions $A$ and $B$ further into three smaller parts.

We emphasize that in the above proof, we did not specify the dimensionality or the geometry of the bipartition. The proof works for any dimension and any bipartition of the system. Thus, it might shed new light on the important challenging problem of proving the entanglement area law for local gapped Hamiltonians in higher dimensions [5,8385], given the possibility that all ground states of these Hamiltonians are perhaps representable by short-range RBMs, although a rigorous proof of this still remains unclear. Intuitively, one can increase the number of hidden neurons to increase the number of local weight parameters. When there are enough free parameters, the corresponding RBMs should be able to represent the ground states of general local gapped Hamiltonians. This would work because of a crucial aspect of our proof-the numbers of hidden neurons and weight parameters are unlimited as long as the connections are finite-ranged.

As shown in our previous work [51], the 1D SPT cluster states, the toric code states in both $2 \mathrm{D}$ and $3 \mathrm{D}$, and the lowenergy excited states with Abelian anyons of the toric code Hamiltonians can all be represented exactly by short-range RBMs with $\mathcal{R}=1$. An immediate corollary of the above theorem is that the entanglement of all these states fulfill an area law. In fact, based on the RBM representation, one can even analytically compute the entanglement spectrum of the 1D SPT cluster state, as we will show in Sec. V. It is important to clarify that although the RBMs are shortranged, their represented quantum states can capture longrange entanglement. The RBM representations of the toric code states (both in 2D and 3D), which have intrinsic topological orders (long-range entangled), are such examples. The area law of short-range RBM states does not imply short-range entanglement. This distinction between short-ranged in the RBM sense and short-ranged in the entanglement sense is an important point.

In 1D, the area-law bound in Eq. (2) gives rise to an interesting relation between the RBM and MPS representations of quantum many-body states. It has been proved that a bounded Rényi entropy of all orders in 1D necessarily guarantees an efficient MPS representation [86] (note that a counterexample does exist if only the von Neumann entropy is bounded [87]). As a result, our area-law results imply that all 1D short-range RBM states can be efficiently described in terms of MPS. However, the validity of the inverse statement is unknown. It would be interesting to find out whether all MPS descriptions with small bond dimensions have efficient RBM representations or not, and if so, what the general procedure is for recasting MPS into RBM states. It would also be interesting to investigate the relations between higher-dimensional RBM states and 
tensor-network states, PEPS, or MERA. Nonetheless, it is worth emphasizing here that the entanglement scaling of RBM states is sharply distinctive from MPS - the maximal entanglement entropy of an $\mathcal{R}$-range RBM state scales linearly with $\mathcal{R}$, whereas a bond-dimension $(\chi)$ MPS has an entanglement entropy scaling as $\log \chi$. This implies that even though a RBM state can be generically converted to a MPS, the parametrization in RBM states is much more efficient for representing highly entangled quantum states.

We remark that our rigorous proof of entanglement area law for short-range RBMs provides a valuable guide for some practical numerical calculations. For instance, in some circumstances, we know that the problem may only involve a small amount (an area-law) entanglement; then, we may use short-range, rather than long-range, RBMs to reduce the number of parameters and consequently speed up the calculations (we have tested this in a numerical experiment of finding the ground state of the transverse-field Ising model via reinforcement learning, and a considerable speedup has indeed been obtained). On the other hand, if the problem to be solved involves large entanglement (such as some quantum criticality or quantum dynamic problems), then short-range RBMs will not necessarily work, and we should choose a long-range RBM to begin with.

\section{VOLUME-LAW ENTANGLEMENT IN LONG-RANGE NEURAL-NETWORK STATES}

In the last section, we proved that all short-range RBM states satisfy an area-law entanglement. What about RBM states with long-range neural connections? From the linearin- $\mathcal{R}$ entanglement-entropy scaling of $\mathcal{R}$-range RBM states derived in the last section, we would anticipate that longrange RBM states could exhibit volume-law entanglement. In this section, we explicitly show that this is indeed true by a rigorous exact construction and a numeric benchmark.

\section{A. Exact construction of maximal volume-law entangled neural-network states}

Here, we analytically construct families of neuralnetwork states with volume-law entanglement. These states are exact and have unified closed-form RBM representations. More strikingly, the RBM representation of these states is surprisingly efficient - the number of nonzero parameters scales only linearly with the system size. We stress that efficient representations of quantum states play a vital role in solving many-body problems, especially when numerical approaches are employed. A prominent example is the advantageous usage of the MPS representation in DMRG [9] (for the ground states), TEBD [19] (for time evolution), and DMRG-X [88] (for highly excited eigenstates of local Hamiltonians deep in the many-body localization region) algorithms. However, the MPS or tensor-network representation is efficient only in describing quantum states with area-law entanglement and thus presents serious practical limitations in solving problems involving volume-law entanglement states. As introduced in the previous section, the construction philosophy of neural-network states is very different from that of MPS or tensor-network states. This gives rise to the possibility for neural networks to efficiently represent quantum states and solve problems with volume-law entanglement. We also stress that our exact results here provide an important anchor point for future theoretical and numerical studies and should have far-reaching implications in the applications of machine-learning techniques in solving currently intractable many-body problems. In Sec. IV C, we indeed use RBMs to solve the ground state (with massive powerlaw entanglement) of a modified Haldane-Shastry model with long-range interactions by using the reinforcement learning.

We first give a 1D example. Let us consider a 1D system of $N$ qubits. The goal is to construct a RBM state with maximal volume-law entanglement entropy. To this end, we introduce a RBM with $N$ visible and $M=\lfloor(3 N) / 2\rfloor-1$ hidden neurons. Here, the floor function $\lfloor x\rfloor$ denotes the largest integer less than or equal to $x$. The weight parameters of $\Phi_{M}(\Xi ; \Omega)$, which characterize the RBM as defined in Eq. (1), are chosen to be

$$
\begin{gathered}
a_{k}=0, \quad \forall k \in[1, N], \\
b_{k}= \begin{cases}-\frac{i \pi}{4} & k \in[1, N-1] \\
\frac{i \pi}{2} & k \in\left[N,\left\lfloor\frac{3 N}{2}\right\rfloor-1\right],\end{cases} \\
W_{k^{\prime} k}= \begin{cases}\frac{i \pi}{4} & \left(k^{\prime}, k\right) \in \mathcal{S} \\
0 & \text { otherwise, }\end{cases}
\end{gathered}
$$

where $\mathcal{S}$ is a set of paired integers defined by $\mathcal{S} \equiv$ $\{(i, j): i \in[1, N-1]$ and $j=i, i+1$ or $i \in[N,\lfloor(3 N) / 2\rfloor-1]$ and $j=i+1-N, i+1-\lceil N / 2\rceil\}$. The ceiling function $\lceil x\rceil$ denotes the smallest integer greater than or equal to $x$. A pictorial illustration of this RBM is shown in Fig. 3. Now, we show that the quantum states described by the above RBM have volume-law entanglement entropy for any contiguous region no larger than half of the system size. To be more precise, we have the following theorem.

Theorem 2.-For a 1D RBM state with weight parameters specified by Eqs. (4)-(6), if we divide the system into two parts $A$ and $B$, with $A$ consisting of the first $l$ $(1 \leq l \leq\lfloor N / 2\rfloor)$ qubits and $B$ the rest, then the corresponding Rényi entropy of $\rho_{A}$ is

$$
S_{\alpha}^{A}=l \log 2, \quad \forall \alpha .
$$

Proof-_As mentioned in Sec. III, since there is no intralayer connection between neurons for a RBM, we can explicitly factor out the hidden variables and rewrite $\Phi_{M}(\Xi ; \Omega)$ in a product form: 


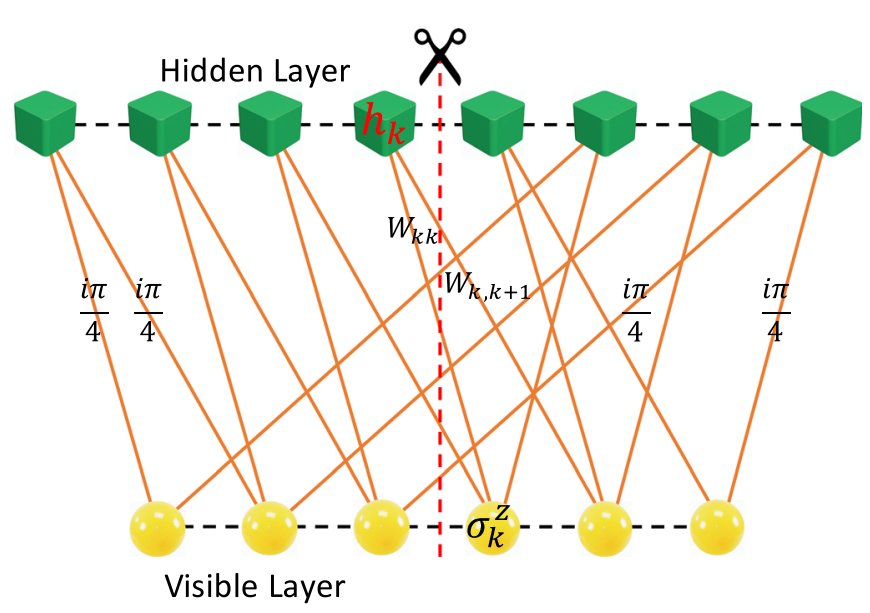

FIG. 3. An illustration of the constructed 1D neural-network state with a maximal volume-law entanglement entropy. This restricted Boltzmann machine has $N$ visible and $\lfloor(3 N) / 2\rfloor-1$ hidden neurons. For $1 \leq k \leq N-1(N \leq k \leq\lfloor(3 N) / 2\rfloor-1)$, the $k$ th hidden neuron is connected to two visible neurons at sites $k$ and $k+1 \quad(k+1-N$ and $k+1-\lceil N / 2\rceil)$ with connection weight parameters equal to $(i \pi) / 4$. The on-site potentials for the visible neurons are chosen to be zero, $a_{k}=0(\forall k)$. For the hidden neurons, $b_{k}$ is chosen as $b_{k}=-[(i \pi) / 4](1 \leq k \leq N-1)$ and $b_{k}=[(\pi i) / 2](N \leq k \leq\lfloor(3 N) / 2\rfloor-1)$. The scissors show a cut of the system into two subsystems $(A$ and $B$ ) with equal sizes, and for this bipartition, the Rényi entropy is $S_{\alpha}^{A}=\lfloor N / 2\rfloor \log 2$, proportional to the system size. This is also the maximal amount of entropy one can have for a system with $N$ qubits.

$\Phi_{M}(\Xi ; \Omega)=\prod_{k=1}^{N} e^{a_{k} \sigma_{k}^{2}} \prod_{k^{\prime}=1}^{M} \Gamma_{k^{\prime}}(\Xi), \quad$ with $\quad \Gamma_{k^{\prime}}(\Xi)=$ $2 \cosh \left(b_{k^{\prime}}+\sum_{k} W_{k^{\prime} k} \sigma_{k}^{z}\right)$. From Eq. (4), $a_{k}=0$ for all $k \in[1, N]$; thus, the first term $\prod_{k=1}^{N} e^{a_{k} \sigma_{k}^{2}}$ simply equals 1 and can be omitted from $\Phi_{M}(\Xi ; \Omega)$. Consequently, the variational wave function $\Phi_{M}(\Xi ; \Omega)$ only depends on the $\Gamma_{k^{\prime}}$ factors, which correspond to the hidden neurons. As shown in Fig. 3, for $k^{\prime} \in[1, N-1], \quad \Gamma_{k^{\prime}}=$ $2 \cosh \left[-(i \pi / 4)+(i \pi / 4)\left(\sigma_{k^{\prime}}^{z}+\sigma_{k^{\prime}+1}^{z}\right)\right]$ connects its corresponding hidden neuron at site $k^{\prime}$ to two nearest-neighbor visible neurons at sites $k^{\prime}$ and $k^{\prime}+1$. Note that $\Gamma_{k^{\prime}}$ has only two possible values: $\Gamma_{k^{\prime}}=-\sqrt{2}$ if $\sigma_{k^{\prime}}^{z}=\sigma_{k^{\prime}+1}^{z}=-1$ and $\Gamma_{k^{\prime}}=\sqrt{2} \quad$ otherwise. For $\quad k^{\prime} \in[N,\lfloor(3 N) / 2\rfloor-1]$, $\Gamma_{k^{\prime}}=2 \cosh \left[(i \pi / 2)+(i \pi / 4)\left(\sigma_{k^{\prime}+1-N}^{z}+\sigma_{k^{\prime}+1-\lceil N / 2\rceil}^{z}\right)\right]$ connects its corresponding hidden neuron at site $k^{\prime}$ to two far-away, separated, visible neurons at sites $k^{\prime}+1-N$ and $k^{\prime}+1-\lceil N / 2\rceil$. Here, $\Gamma_{k^{\prime}}$ vanishes if $\sigma_{k^{\prime}+1-N}^{z}=-\sigma_{k^{\prime}+1-\lceil N / 2\rceil}^{z} \quad$ and $\quad \Gamma_{k^{\prime}}=2 \quad\left(\Gamma_{k^{\prime}}=-2\right) \quad$ if $\sigma_{k^{\prime}+1-N}^{z}=\sigma_{k^{\prime}+1-\lceil N / 2\rceil}^{z}=-1 \quad\left(\sigma_{k^{\prime}+1-N}^{z}=\sigma_{k^{\prime}+1-\lceil N / 2\rceil}^{z}=1\right)$. These features of the $\Gamma_{k^{\prime}}$ factors are crucial in the following proof of Eq. (7).

For convenience, we define two sets of integers: $\mathcal{B}_{1}=$ $\{j: N+1-\lceil N / 2\rceil \leq j \leq N+l+1-\lceil N / 2\rceil\}$ and $\mathcal{B}_{2}=$ $\{j: l+1<j<N+1-\lceil N / 2\rceil$ or $N+l+1-\lceil N / 2\rceil<j \leq N\}$. We note that $B=\mathcal{B}_{1} \cup \mathcal{B}_{2}$. By using the features of the $\Gamma_{k^{\prime}}$ factors discussed above, the RBM state reduces to
$|\Psi(\Omega)\rangle \equiv \sum_{\Xi_{A \cup B_{2}}} \chi_{\Xi_{A \cup \mathcal{B}_{2}}}\left|\Xi_{A}\right\rangle\left|\Xi_{\mathcal{B}_{1}}: \Xi_{\mathcal{B}_{1}}=\Xi_{A}\right\rangle\left|\Xi_{\mathcal{B}_{2}}\right\rangle$, where $\chi_{\Xi_{A \cup B_{2}}}= \pm C$ is a coefficient depending on $\Xi_{A \cup B}$, with $C$ a positive constant. By tracing out the degrees of freedom in region $B$ and putting back the normalization constant, we obtain the reduced density matrix $\rho_{A}=\mathbf{I} / 2^{l}$, with $\mathbf{I}$ the identity matrix of dimension $2^{l} \times 2^{l}$. This completes the proof.

It is worthwhile to mention that the subregion $A$ does not necessarily have to be at the left end. In fact, $A$ can be any contiguous region of length $l$, and Eq. (7) still remains valid, although the details of the proof would change slightly in this situation. We choose $A$ to be at the left end just for convenience. In the limit $N \rightarrow \infty$, for any contiguous region, its entanglement entropy scales linearly with the size of the region-a volume-law entanglement.

For the 2D case, we can construct volume-law-entangled RBM states in a similar manner. We consider a system of $N$ qubits living on an $L_{x} \times L_{y}$ square lattice denoted as $\Lambda$. We assume $L_{x}$ and $L_{y}$ are even integer numbers, for simplicity (one can use the floor and ceiling functions to deal with the case of odd numbers, but the notations will be more cumbersome). We label each vertex of the lattice by a pair of indices $\left(k_{x}, k_{y}\right)\left(1 \leq k_{x} \leq L_{x}\right.$ and $\left.1 \leq k_{y} \leq L_{y}\right)$ and attach a qubit $N=L_{x} \times L_{y}$ to it. We construct a RBM with $N$ visible and $\frac{5}{2} L_{x} L_{y}-L_{x}-L_{y}$ hidden neurons. The hidden neurons are divided into three groups. The first (second) group, denoted by $\mathcal{X}(\mathcal{Y})$, has $\left(L_{x}-1\right) \times L_{y}$ $\left[L_{x} \times\left(L_{y}-1\right)\right]$ neurons that connect nearest visible neurons along the $x(y)$ direction. The third group, denoted by $\mathcal{Z}$, contains $L_{x} \times \frac{1}{2} L_{y}$ hidden neurons that connect visible neurons nonlocally. One can draw an analogy with the 1D example: The neurons in groups $\mathcal{X}$ and $\mathcal{Y}$ connect nearest visible neurons, and they correspond to the first $N-1$ hidden neurons in the 1D case; similarly, those in group $\mathcal{Z}$ correspond to the remaining ones. The hidden neurons in $\mathcal{X}, \mathcal{Y}$, and $\mathcal{Z}$ are labeled by $\mathbf{x}=\left(x_{1}, x_{2}\right), \mathbf{y}=\left(y_{1}, y_{2}\right)$, and $\mathbf{z}=\left(z_{1}, z_{2}\right)$, respectively. Following the $1 \mathrm{D}$ example, the weight parameters can be chosen as

$$
\begin{aligned}
a_{k_{x} k_{y}} & =0, \quad \forall\left(k_{x}, k_{y}\right) \in \Lambda, \\
b_{\mathbf{x}}^{(\mathcal{X})} & =b_{\mathbf{y}}^{(\mathcal{Y})}=-\frac{i \pi}{4}, \quad b_{\mathbf{z}}^{(\mathcal{Z})}=\frac{i \pi}{2}, \\
W_{\mathbf{x} / \mathbf{y} / \mathbf{z} ; k_{x} k_{y}}^{(\mathcal{X} / \mathcal{Z} / \mathcal{Z})} & = \begin{cases}\frac{i \pi}{4} & \left(\mathbf{x} / \mathbf{y} / \mathbf{z} ; k_{x}, k_{y}\right) \in \mathcal{S}_{2 D}^{(\mathcal{X} / \mathcal{Y} / \mathcal{Z})} \\
0 & \text { otherwise },\end{cases}
\end{aligned}
$$

where $\mathcal{S}_{2 D}^{(\mathcal{X})}, \mathcal{S}_{2 D}^{(\mathcal{Y})}$, and $\mathcal{S}_{2 D}^{(\mathcal{Z})}$ are the three sets that specify the connections between the visible neurons and the three groups of hidden neurons. They are defined as $\mathcal{S}_{2 D}^{(\mathcal{X})} \equiv\left\{\left(\mathbf{x} ; k_{x}, k_{y}\right): k_{x}=x_{1}, x_{1}+1 ; k_{y}=x_{2}\right\}, \quad \mathcal{S}_{2 D}^{(\mathcal{Y})} \equiv$ $\left\{\left(\mathbf{y} ; k_{x}, k_{y}\right): k_{x}=y_{1} ; k_{y}=y_{2}, y_{2}+1\right\}, \quad \mathcal{S}_{2 D}^{(\mathcal{Z})} \equiv\left\{\left(\mathbf{z} ; k_{x}, k_{y}\right):\right.$ $\left(k_{x}, k_{y}\right)=\left(z_{1}, z_{2}\right),\left(f\left(z_{1}\right), z_{2}+\left(L_{y} / 2\right)\right\}$, with $f\left(z_{1}\right)=$ $z_{1}+\left(L_{x} / 2\right)$ if $1 \leq z_{1} \leq\left(L_{x} / 2\right)$ and $f\left(z_{1}\right)=z_{1}-\left(L_{x} / 2\right)$ 
if $\left(L_{x} / 2\right)<z_{1} \leq L_{x}$. Following the proof of theorem 2, it is straightforward to verify that the entanglement entropy for any small regular contiguous subregion $A$ scales linearly with the volume of $A$ and is maximal, $S_{\alpha}^{A}=N_{A} \log 2$. Here, $N_{A}$ denotes the number of qubits inside region $A$.

We mention that similar constructions carry over to higher dimensions straightforwardly. For a system defined on a simple cubic lattice in $d$ dimensions with $N=L^{d}$ qubits, our construction requires $M=[(2 d+1) / 2] L^{d}-$ $d L^{d-1}$ hidden neurons and $3 M$ nonzero weight parameters. Both the number of hidden neurons and the number of parameters scale only linearly with the system size. In contrast, if we express these RBM states in terms of MPS or tensor networks, the bond dimension will grow exponentially with the system size, and the problem quickly becomes intractable. This result explicitly demonstrates a unique advantage of RBMs in representing quantum manybody states with massive entanglement.

\section{B. Entanglement benchmarking}

For a general RBM state with long-range connections, the entanglement entropy cannot be calculated analytically. We thus resort to numerical simulations. We study the entanglement properties of RBM states with random weight parameters. We consider a $1 \mathrm{D}$ system with $N$ qubits. The corresponding RBM has $N$ visible and $M$ hidden neurons, with the weight parameters chosen randomly and independently. For each random sample, we numerically calculate the coefficients for all possible spin configurations (there are $2^{N}$ configurations) and normalize them to obtain the corresponding quantum state in the computational basis. We then make an equal bipartition and calculate the reduced density matrix $\rho_{A}$ for the $A$ subsystem. We diagonalize $\rho_{A}$ to compute the desired entanglement entropy and spectrum. The number of samples used for numerics ranges from $10^{6}(N=6)$ to $10^{3}$ $(N=22)$. We mention that although we focus only on 1D systems, some entanglement features discovered here should carry over to higher dimensions as well. Extensive higher-dimensional RMB-based numerics are left for future studies.

In Fig. 4(a), we plot the averaged entanglement entropy scaling with different system sizes. When $\gamma$ is small $(\gamma=1$, 2,3 ), we find that the averaged entanglement entropy scales linearly with the system size-a volume law (this is another indication that entanglement is not the limiting factor for the RBMs in representing quantum many-body states). Here, $\gamma=M / N$ denotes the ratio between the number of hidden and visible neurons. However, when $\gamma$ increases, the entanglement apparently bends downwards and seems to saturate at large $N$. This result seems surprising at first sight because an increase of $\gamma$ means an increase in the number of connections between visible neurons, and intuitively, the entanglement should increase as well. In fact, the bending of the curve at large $\gamma$ may be understood by looking at the original RBM representation in Eq. (1). Since we choose $W_{k k^{\prime}}$ randomly, on average $\Phi_{M}(\Xi ; \Omega)$ will become less and
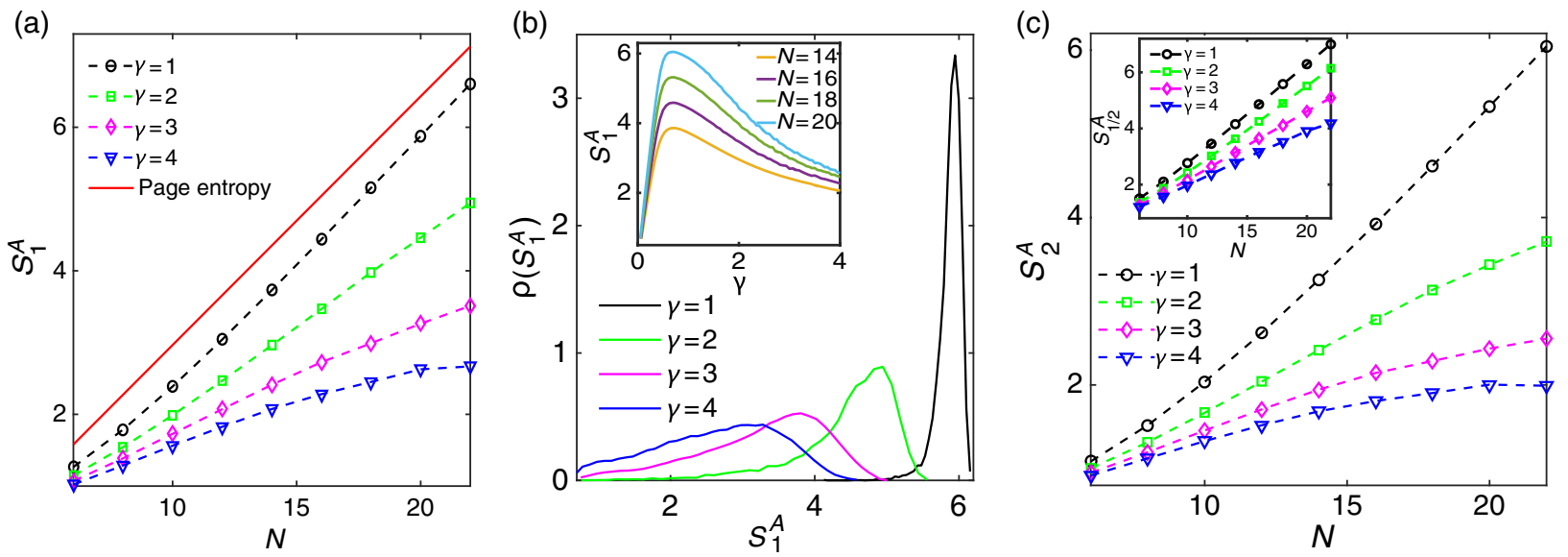

FIG. 4. Entanglement scaling and distributions of neural-network states with random weight parameters. (a) The averaged von Neumann entropy $S_{1}^{A}$. For small $\gamma\left(\gamma=M / N\right.$ denoting the ratio between the number of hidden and visible neurons), $S_{1}^{A}$ scales linearly with the system size, indicating a volume-law entanglement. However, $S_{1}^{A}$ tends to saturate when $\gamma$ becomes large $(\gamma=4)$. Moreover, for all $\gamma$ values studied, $S_{1}^{A}$ deviates significantly from the Page value for the entanglement entropy averaged over random pure states. (b) The entanglement distribution of $S_{1}^{A}$ for different $\gamma$. The peak shifts to the left as $\gamma$ increases, which is consistent with the observation that the averaged $S_{1}^{A}$ decreases as we increase $\gamma$. Here, the system size is chosen to be $L=20$, and we have used $10^{4}$ random samples. The inset shows $S_{1}^{A}$ as a function of $\gamma$ for different system sizes. Note that $S_{1}^{A}$ reaches its maximal value at $\gamma^{*} \approx 0.7$. However, even this maximal value of $S_{1}^{A}$ is still noticeably smaller than the Page value for random states. (c) The scaling of the Rényi entropy with the system size. The second-order Rényi entropy $S_{2}^{A}$ behaves similarly to the von Neumann entropy $S_{1}^{A}$. The inset shows the results for $S_{1 / 2}^{A}$. For simplicity, we have chosen the on-site potentials $a_{k}(k \in[1, N])$ and $b_{k^{\prime}}\left(k^{\prime} \in[1, M]\right)$ to be zero. The connection weight parameters $W_{k k^{\prime}}$ are complex numbers randomly drawn from uniform distributions with $\operatorname{Re}\left(W_{k k^{\prime}}\right) \in[-3 / N, 3 / N]$ and $\operatorname{Im}\left(W_{k k^{\prime}}\right) \in[-\pi, \pi]$. 
less dependent on the spin configuration $\Xi$ as $\gamma$ increases. In other words, in the represented many-body quantum wave function, the difference between the coefficients of each component becomes smaller and smaller. Thus, the state becomes closer and closer to a product state, and therefore, the entanglement decreases. This result is further justified in the inset of Fig. 4(b), where the von Neumann entropy $S_{1}^{A}$ is shown as a function of $\gamma$ for different system sizes. From this figure, we see that $S_{1}^{A}$ reaches its maximal value at a critical $\gamma^{*} \approx 0.7$, independent of system size. When $\gamma>\gamma^{*}$, $S_{1}^{A}$ decreases as we increase $\gamma$. It is also worthwhile to mention that when we fix $M$ as a finite number $(\gamma \rightarrow 0$ in the thermodynamic limit $N \rightarrow \infty$ ), then $S_{1}^{A}$ is upper bounded by $M \log 2$, regardless of the system size. This result can be understood heuristically by imagining all the hidden neurons being grouped into subsystem $B$; then, subsystem $A$ can only have, at most, $2^{M}$ degrees of freedom that are entangled with $B$. Consequently, $S_{1}^{A}$ is bounded by $M \log 2$. This explains the numerical observation in the inset of Fig. 4(b), that for $M=1$ (smallest $\gamma$ ), $S_{1}^{A} \approx \log 2$ independent of the system size.

In order to compare the RBM states with random parameters with generic random pure states, we also calculate the so-called Page entropy [89], which is the averaged entanglement entropy over pure states drawn randomly from the entire Hilbert space of the system. The Page entropy provides an estimate for entanglement in extended thermal states [90] and has been widely used in the context of quantum chaos [91], black hole information [92,93], and many-body localization [94,95]. From the random matrix theory, it can be computed as $S_{\text {Page }}=-\left[\left(d_{A}-1\right) / 2 d_{B}\right]+\sum_{j=d_{B}+1}^{d_{A} d_{B}}(1 / k)$, where $d_{A}$ and $d_{B}$ denote the Hilbert space dimensions of subsystems $A$ and $B$, respectively [89]. An interesting observation in Fig. 4(a) and the inset of Fig. 4(b) is that the entanglement entropy is always smaller than the Page entropy for all $\gamma$. This implies that the pattern of entanglement for the RBM states with random parameters is distinct from that of random pure states, which is consistent with the fact that the RBM states live in a very small restricted subspace of the entire Hilbert space. This also indicates that a random state in the Hilbert space is probably not well described by a RBM efficiently. In Fig. 4(b), we plot the entanglement distribution for different $\gamma$. We find that, as $\gamma$ increases, the distribution becomes broader, and the density peak shifts towards smaller values. This result is in agreement with the observation in Fig. 4(a) that the entanglement decreases as $\gamma$ increases. Figure 4(c) shows the results for the Rényi entropy of orders $\alpha=2$ and $\alpha=1 / 2$. As expected, $S_{2}$ behaves very similarly to $S_{1}$. For $S_{1 / 2}$, we find a similar volume-law scaling of entanglement, but the bending feature does not show up at $\gamma=4$ because of finite-size effects.

The entanglement entropy studied above provides a wealth of information about the data structure of the RBM states. However, as has been realized in a number of different physical contexts, the entanglement entropy cannot capture the full entanglement structure of the system [96-101]. Much greater information can be extracted from the entanglement spectrum. In order to obtain a more comprehensive understanding of the data structure of the RBM states with random weight parameters, we have therefore also calculated their entanglement spectra and the entanglement Hamiltonian level statistics. In Fig. 5(a), we plot the averaged entanglement spectrum for different $\gamma$.
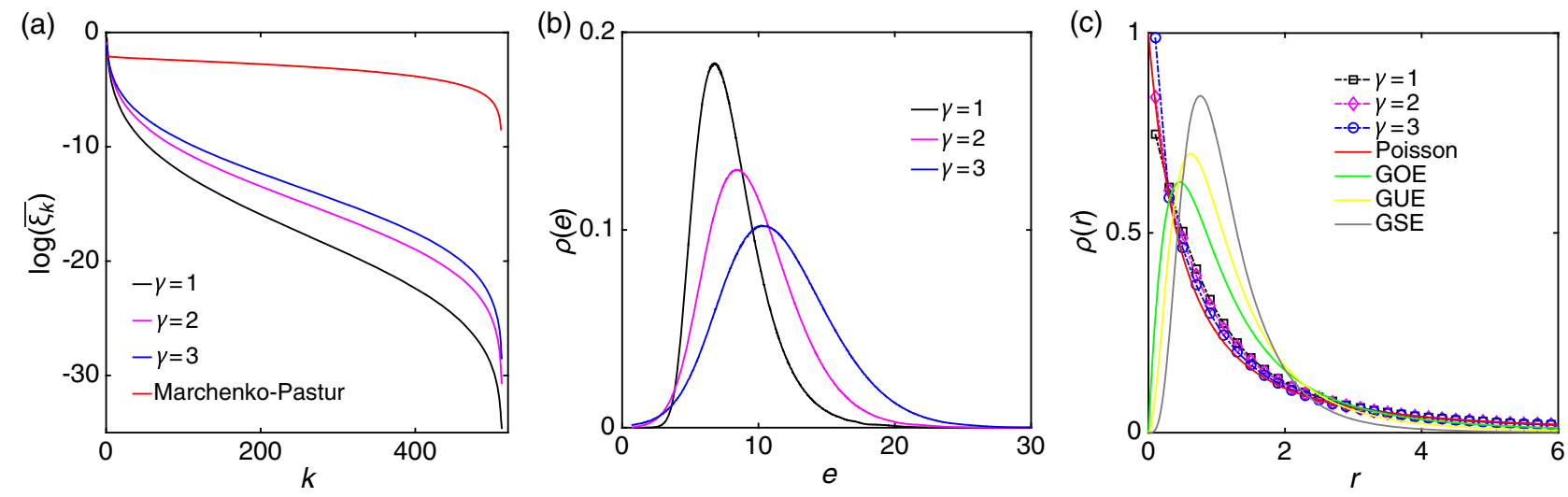

FIG. 5. Entanglement spectrum and level statistics for neural-network states with random weight parameters. Here, the lattice size is chosen to be $L=20$, and we have used $10^{4}$ random samples. The random parameters are drawn from the same distribution as specified in Fig. 4. (a) Averaged entanglement spectrum with different $\gamma$. Here, $\xi_{k}$ denotes the $k$ th eigenvalue of $\rho_{A}$ (the eigenvalues are arranged in descending order). The red line denotes the spectrum of a completely random state (derived from a Marchenko-Pastur distribution). It is evident that the entanglement spectra of the restricted-Boltzmann-machine states with random parameters are completely distinct from the Marchenko-Pastur distribution, indicating that their corresponding entanglement Hamiltonians are very different from Wishart matrices. (b) Density of states for the entanglement Hamiltonians. Here, $e$ denotes the eigenspectrum of $H_{\text {ent }}$. When increasing $\gamma$, the distribution of the eigenvalues broadens, and the peak shifts rightwards. (c) Distributions of the ratios of consecutive spacings for the entanglement spectrum. These distributions follow a Poisson law and differ significantly from the predictions of GOE, GUE, or GSE. 
We find that the entanglement spectrum for the RBM states is completely different from the Marchenko-Pastur distribution derived from random matrix theory [102]. More specifically, the Marchenko-Pastur distribution describes the asymptotic average density of eigenvalues of a Wishart matrix (a matrix of the form $Y=X X^{\dagger}$, with $X$ a random rectangular matrix). It was shown recently in Ref. [97] that the entanglement spectrum of highly excited eigenstates in the delocalized phase bears a two-component structure: (i) a universal part that is associated with random matrix theory, i.e., a universal tail that follows the MarchenkoPastur distribution and thus is model independent, and (ii) a model-dependent nonuniversal part that dominates the weights in the spectrum. In the localized phase, the universal part of the spectrum disappears in the thermodynamic limit, leaving only the nonuniversal part that leads to an area-law scaling of the entanglement entropy. In our case for the RBM states with random weight parameters, the universal part disappears completely even for a system size as small as $N=20$. In this sense, these RBM states are less random than the highly excited eigenstates in both the delocalized and localized phases. This further shows that, although these RBM states obey a volume law of entanglement entropy on average, they are living in a very restricted subspace of the entire Hilbert space. In Fig. 5(b), we plot the density of states for the entanglement Hamiltonian of these RBM states. We find that a broader distribution shows up as we increase $\gamma$, and the peak moves to the right, which is consistent with the surprising results from Fig. 4 (i.e., entanglement decreases as $\gamma$ increases).

Another quantity that is also useful in understanding the data structure of the RBM states is the adjacent gap ratio $r$ defined as $r_{n}=\left[\min \left(\delta_{n}, \delta_{n-1}\right) / \max \left(\delta_{n}, \delta_{n-1}\right)\right]$, with $\delta_{n}$ the level spacing between the $n$th and the $(n-1)$ th eigenstates of the entanglement Hamiltonian. We note that the importance of the distribution of $r$ has been broadly appreciated in various contexts. In quantum chaos [103], it is argued that whereas the level statistics for integrable quantum Hamiltonians obeys a Poisson law [104], the case for Hamiltonians with chaotic dynamics must follow one of the three classical ensembles from random matrix theory [105], namely, the Gaussian orthogonal ensemble (GOE), the Gaussian unitary ensemble (GUE), and the Gaussian symplectic ensemble (GSE). These three ensembles correspond to Hermitian random matrices, with entries being independently distributed random real, complex, and quaternionic variables, respectively [106]. In many-body localization, it is generally believed (recently verified by extensive numerical calculations) that Hamiltonians in the delocalized and localized regions manifest, respectively, GOE (or GUE) and Poisson level statistics [68]. The level statistics of the entanglement Hamiltonians in this context has also been studied recently in Ref. [98]. It was shown that, in the thermal phase, the entanglement spectrum shows level statistics in agreement with predictions from random matrix theory and is governed by the same random matrix ensemble as the energy spectrum. However, in the many-body localized phase, the entanglement spectrum shows a semi-Poisson distribution, in contrast with the energy spectrum following a Poisson law. For the RBM states with random weight parameters studied in this section, we find that their entanglement spectra follow a Poisson distribution, as shown in Fig. 5(c). The averaged value of $r_{n}$ (over $10^{4}$ random samples) with $N=20$ and $\gamma=3$ equals 0.378 , which is in good agreement with the Poisson predicted value $2 \ln 2-1 \approx 0.386$ [107]. The small deviation could be attributed to finite-size effects. Thus, these RBM states are distinct from the eigenstates of Hamiltonians in either the delocalized or localized phases on average. In addition, we also remark that the Poisson behavior and the lack of a universal part in the entanglement spectra of these RBM states imply that they are not irreversible-namely, there exists an efficient algorithm to completely disentangle these states [52]. Finding the disentangling algorithm would provide some insight into the nature of neural-network quantum states and is an interesting topic for future investigation.

\section{Reinforcement learning of ground states with power-law entanglement}

The above discussion shows that, unlike MPS or tensornetwork states, entanglement is not the limiting factor for the efficiency of the RBM representation. As an important consequence, RBM might be capable of solving some quantum many-body problems where massive entanglement is involved. To demonstrate this unprecedented power, in this section, we consider the problem of finding the ground state (with power-law entanglement) of a spin$1 / 2$ Hamiltonian with long-range interaction, through a reinforcement-learning scheme [22,53]. We consider $N$ spin-1/2 particles living on a ring (see Fig. 6) with a modified Haldane-Shastry [54,55] Hamiltonian given by

$$
H_{\mathrm{MHS}}=\sum_{j<k}^{N} \frac{1}{d_{j k}^{2}}\left(-\hat{\sigma}_{i}^{x} \hat{\sigma}_{j}^{x}-\hat{\sigma}_{i}^{y} \hat{\sigma}_{j}^{y}+\hat{\sigma}_{i}^{z} \hat{\sigma}_{j}^{z}\right),
$$

where $\quad d_{i j}=(N / \pi)|\sin [\pi(j-k) / N]|$ is the so-called "chord distance." Since it has long-range interactions with a power-law decaying strength, we expect its ground state to have power-law entanglement. Although a rigorous proof is still lacking and seems very hard to obtain, we can verify the entanglement power law numerically. In Fig. 7(a), we plot the von Neumann entropy for the ground state of $H_{\mathrm{MHS}}$ calculated from exact diagonalization (ED). We find that it indeed has an excellent power-law fit with the system size.

We now show that RBM is capable of faithfully and efficiently representing the ground state of $H_{\mathrm{MHS}}$, and the representing RBM can be efficiently obtained via 


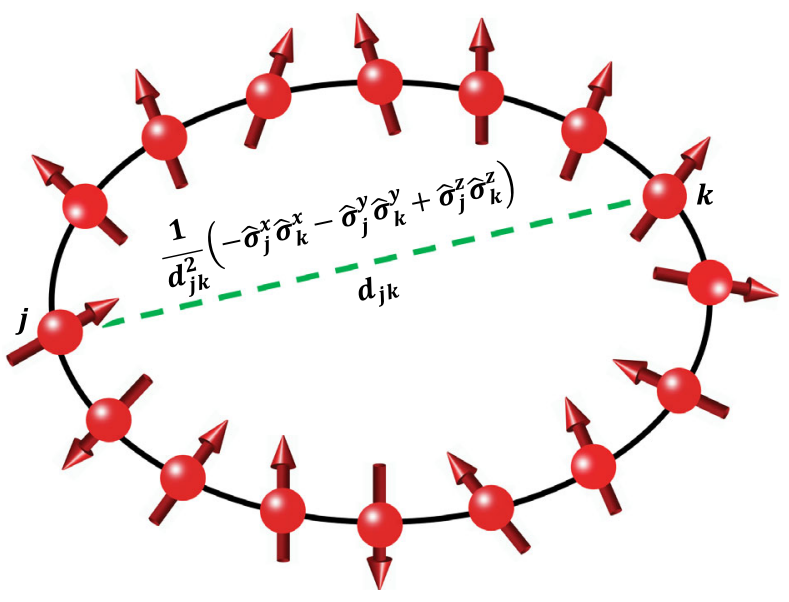

FIG. 6. The modified Haldane-Shastry model. The $N$ spin- $-\frac{1}{2}$ particles form an equally spaced lattice on a ring. Each spin is interacting with all the other spins. The interaction between spin $j$ and $k$ is of Heisenberg $X X Z$ type, and its strength is inversely proportional to the square of the chord distance $d_{j k}$ [see Eq. (8)]. The ground state of this model has power-law entanglement and can be calculated with a restricted Boltzmann machine through reinforcement learning.

reinforcement learning, despite the fact that the ground state has a large amount of entanglement entropy. Since the Hamiltonian has a lattice translation symmetry, we can use this symmetry to reduce the number of variational parameters, and for integer hidden-variable density $(\gamma=1,2, \ldots)$, the weight matrix takes the form of feature filters $W_{j}^{(f)}$ with $f \in[1, \gamma]$, as described in Ref. [22]. In Fig. 7(b), we plot the different spin correlations obtained via reinforcement learning, for small system sizes. We compare the RBM result with that from exact diagonalization. As shown in this figure, the RBM result matches the ED result very well. The accuracy of the RBM result can be improved by increasing $\gamma$ and the number of iterations in the training process. In Fig. 7(c), we show the feature maps after a typical reinforcement learning process with $\gamma=4$ and $N=20$. The accuracy of the trained RBM can be quanti-

fied by the relative error on the ground-state energy $\epsilon_{\text {rel }}=$ $\left|\left(E_{0}^{(\mathrm{RBM})}-E_{0}^{\mathrm{ED}}\right) / E_{0}^{\mathrm{ED}}\right|[22]$. For the parameters shown in Fig. 7(c), we find $\epsilon_{\text {rel }} \sim 10^{-5}$. We then calculate the correlation functions and ground-state energy density for larger system sizes, which are far beyond the capability of the ED technique. We plot some of the results for $N=100$ in Fig. 7(d). We find that the correlation $\left\langle\hat{\sigma}_{1}^{z} \hat{\sigma}_{1+j}^{z}\right\rangle$ has a sharp jump at $j=2$, which is also obtained in our ED calculations for smaller system sizes, as shown in Fig. 7(b).

We remark that the DMRG/MPS-based simulations are particularly challenging for the above problem and would presumably require a substantially larger number of variational parameters than the RBM approach $[9,108]$. In this regard, the reinforcement-learning-based RBM technique has apparent advantages when large entanglement and
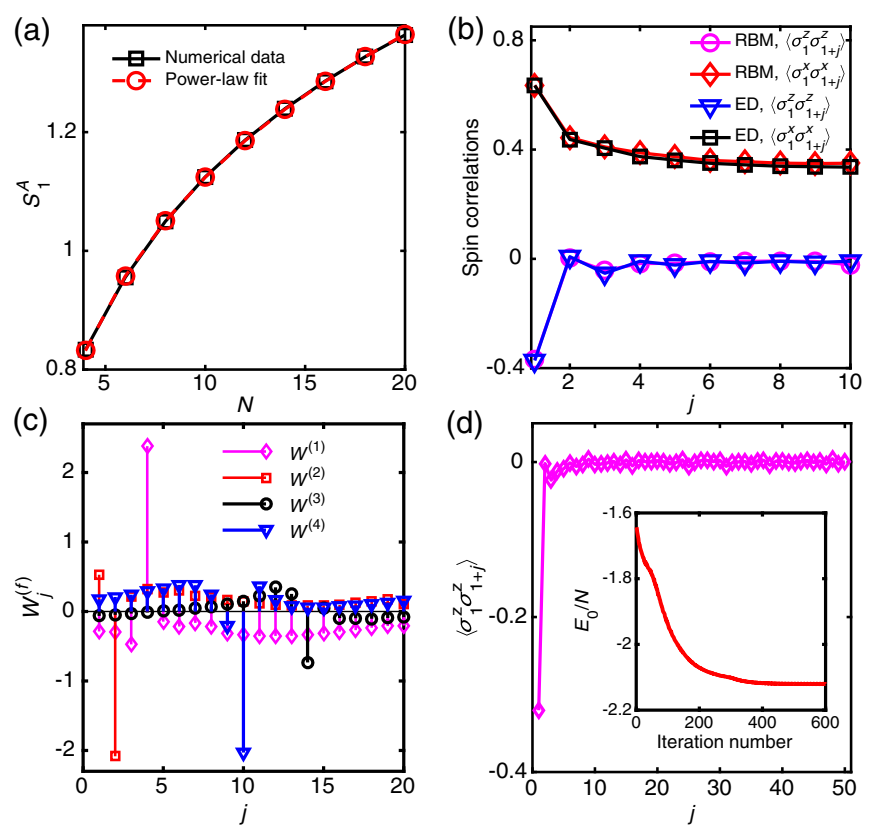

FIG. 7. Reinforcement learning of the ground state of the modified Haldane-Shastry model with power-law entanglement. (a) The von Neumann entropy calculated by exact diagonalization (ED) of the Hamiltonian with different system sizes. It has an excellent power-law fit: $S_{1}^{A} \sim 2.473 N^{0.106}-2.033$. Here, the entropy is calculated from an equal bipartition of the system. (b) Spin correlations for small system sizes calculated by ED and a restricted Boltzmann machine, respectively. (c) The learned feature maps for representing the ground state with a restricted Boltzmann machine. In panels (b) and (c), the hidden-unit density $\gamma=4$, and the system size is fixed to $N=20$. (d) Reinforcement learning of the spin correlations and ground-state energy density for larger system size $N=100$. The inset shows the variational energy density as a function of the iteration number of the learning process. As the iteration number increases, the energy converges smoothly to an asymptotic value around $E_{0} / N \approx-2.12$.

long-range interactions are involved. Moreover, as pointed out in Ref. [22], the RBM approach also works in higher dimensions and for dynamical problems. We also mention that $H_{\mathrm{MHS}}$ might be realized with trapped ions in a ring geometry $[109,110]$. Thus, the numerically calculated correlations could be experimentally verified in the future.

\section{AN ANALYTICAL RBM RECIPE FOR CALCULATING ENTANGLEMENT}

In Sec. III, we proved that all short-range RBM states obey an area-law entanglement. Can we calculate the entanglement entropy and spectrum analytically? For a general many-body state, this is an outstanding challenge, especially for a system with a finite size. In fact, most of the past works focus on the thermodynamic limit and compute entanglement entropy asymptotically. The methods used often involve complicated mathematics [4]. For instance, 
the Fisher-Hartwig formula has been used to evaluate the asymptotic behavior of the entanglement entropy for the critical $X X$ model and other isotropic models [111-114]. Another notable approach is the use of conformal field theory, where some universal properties of entanglement entropy have been established for critical $(1+1)$ dimensional systems [115-117]. For calculating the exact entanglement entropy of a finite system, the quotient group method has been used to calculate the entropy of an arbitrary bipartition of the 2D toric code states $[118,119]$. Here, however, we introduce an alternative approach and show that the RBM representation would also be helpful in the analytical calculation of the entanglement entropy and spectrum. As an example, we consider the 1D SPT cluster state $|\Psi\rangle_{\text {cluster, }}$, which is the ground state of the cluster Hamiltonian $H_{\text {cluster }}$ defined on a 1D lattice with a periodic boundary condition: $H_{\text {cluster }}=$ $-\sum_{k=1}^{N} \hat{\sigma}_{k-1}^{z} \hat{\sigma}_{k}^{x} \hat{\sigma}_{k+1}^{z}$. This state is a topological state protected by $Z_{2} \times Z_{2}$ symmetry [120]. It serves as a simple toy model for studying SPT phases and has important applications in measurement-based quantum computation [121123]. An exact and efficient RBM representation of the 1D cluster state has been found in Ref. [51]. This representation has $N$ hidden neurons, with each one connecting only locally to the visible neurons within a distance of 1 . The weight parameters are specified as

$a_{k}=0, \quad b_{k}=\frac{i \pi}{4}, \quad W_{k j}= \begin{cases}i \omega_{\mu} & \text { if }|k-j|=1 \\ 0 & \text { otherwise }\end{cases}$

where $\omega_{\mu} \mathrm{s}(\mu=1,0,-1)$ are positive real numbers given by $\left(\omega_{1}, \omega_{0}, \omega_{-1}\right)=(\pi / 4)(2,3,1)$. In the product form, the normalized $1 \mathrm{D}$ cluster state reads

$$
|\Psi\rangle_{\text {cluster }}=\sum_{\Xi} \prod_{k^{\prime}=1}^{N} \Gamma_{k^{\prime}}(\Xi)|\Xi\rangle,
$$

where the $\Gamma_{k^{\prime}}$ factor only depends on the configurations of three nearest visible neurons $\Gamma_{k^{\prime}}(\Xi)=\Gamma_{k^{\prime}}\left(\sigma_{k^{\prime}-1}^{z}, \sigma_{k^{\prime}}^{z}, \sigma_{k^{\prime}+1}^{z}\right)=$ $\cos \left[(\pi / 4)\left(1+2 \sigma_{k^{\prime}-1}^{z}+3 \sigma_{k^{\prime}}^{z}+\sigma_{k^{\prime}+1}^{z}\right)\right]$ (note that we returned the normalization constant and rescaled all the $\Gamma_{k^{\prime}}$ factor by $1 / 2$ ).

In order to study its entanglement properties, we consider an arbitrary bipartition of the system into two parts, $A$ and $B$, and we aim to calculate the entanglement entropy and spectrum of subsystem $A$ analytically. For convenience, we further divide the subregion $A(B)$ into two parts, $A_{1}$ and $A_{2}\left(B_{1}\right.$ and $\left.B_{2}\right)$, with $A_{2}\left(B_{2}\right)$ containing only four sites, $\alpha_{1}$, $\alpha_{2}, \alpha_{3}$, and $\alpha_{4}\left(\beta_{1}, \beta_{2}, \beta_{3}\right.$, and $\left.\beta_{4}\right)$, as shown in Fig. 8. Using the fact that the RBM is short-ranged and $\Gamma_{k^{\prime}}(\Xi)= \pm(\sqrt{2} / 2)$, we can rewrite $|\Psi\rangle_{\text {cluster }}$ in the following form, where the subregions $A$ and $B$ appear explicitly,

$$
|\Psi\rangle_{\text {cluster }}=\frac{1}{4} \sum_{\Xi_{A_{2} \cup B_{2}}} \Gamma_{\alpha_{2}} \Gamma_{\alpha_{4}} \Gamma_{\beta_{2}} \Gamma_{\beta_{4}}\left|\Psi_{A}\right\rangle\left|\Psi_{B}\right\rangle
$$

with $\left|\Psi_{A}\right\rangle=\left|\Psi_{A}\left(\Xi_{A_{2}}\right)\right\rangle=2 \sum_{\Xi_{A_{1}}} \Gamma_{\alpha_{1}} \Gamma_{\alpha_{3}} \prod_{k^{\prime} \in A_{1}} \Gamma_{k^{\prime}}\left(\Xi_{A}\right)\left|\Xi_{A}\right\rangle$ and $\left|\Psi_{B}\right\rangle=\left|\Psi_{B}\left(\Xi_{B_{2}}\right)\right\rangle=2 \sum_{\Xi_{B_{1}}} \Gamma_{\beta_{1}} \Gamma_{\beta_{3}} \prod_{k^{\prime} \in B_{1}} \Gamma_{k^{\prime}}\left(\Xi_{B}\right)\left|\Xi_{B}\right\rangle$. Equation (11) is crucial in calculating the entanglement entropy and spectrum. Compared with Eq. (10), it contains only $2^{8}$, rather than $2^{N}$, terms in the summation. Noting that $\left|\Psi_{A}\right\rangle\left(\left|\Psi_{B}\right\rangle\right)$ only depends on the spin configurations within subregion $A_{2}\left(B_{2}\right)$ and $\left\langle\Psi_{A}\left(\Xi_{A_{2}}\right) \mid \Psi_{A}\left(\Xi_{A_{2}}^{\prime}\right)\right\rangle=\delta_{\Xi_{A_{2}}, \Xi_{A_{2}}^{\prime}}$ one can perform a unitary transformation $U_{A}\left(U_{B}\right)$ within subregion $A(B)$ to rotate the basis of the Hilbert space $H_{A}\left(H_{B}\right)$ of $A(B)$. Note that this rotation will not affect the entanglement entropy and spectrum. In the new basis, $\left|\Psi_{A}\left(\Xi_{A_{2}}\right)\right\rangle\left[\left|\Psi_{B}\left(\Xi_{B_{2}}\right)\right\rangle\right]$ is just a basis vector of $H_{A}\left(H_{B}\right)$. By tracing out the degrees of freedom in subregion $B$ and plugging in the parameter values in Eq. (9), we find a very simple expression for the reduced density matrix $\rho_{A}$ in the new basis,

$$
\rho_{A}=M_{1} \oplus M_{1} \oplus M_{2} \oplus M_{2} \oplus \mathbf{0}
$$

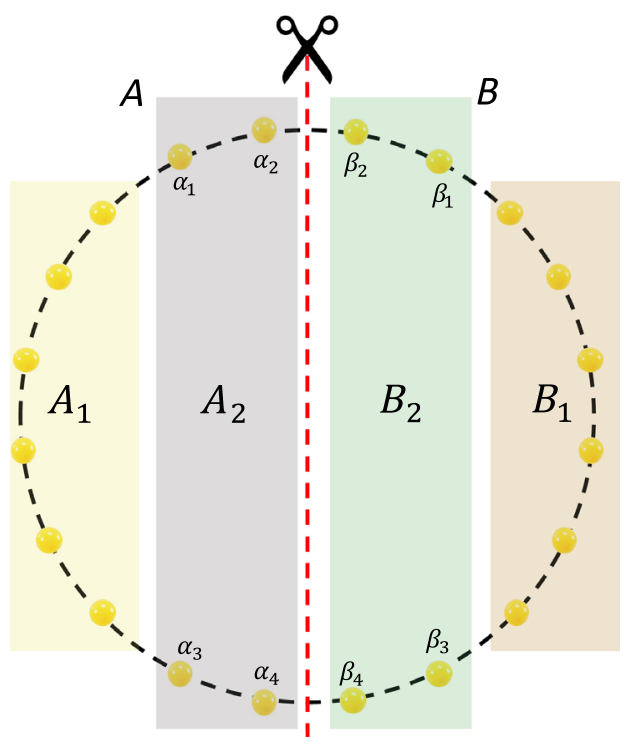

FIG. 8. A sketch for analytically computing the entanglement entropy and spectrum of the 1D symmetry-protected-topological cluster state, through the corresponding exact short-range restricted-Boltzmann-machine representation. The scissors show a cut of the system into two subsystems $A$ and $B$. We calculate the entanglement entropy and spectrum from the reduced density matrix $\rho_{A}$. In order to conveniently explore the short-range feature of the restricted-Boltzmann machine, we further divide $A(B)$ into $A_{1}$ and $A_{2}\left(B_{1}\right.$ and $\left.B_{2}\right)$. It is important that the $\Gamma_{k^{\prime}}$ factors in subregion $A_{1}\left(B_{1}\right)$ are independent of spin configurations in $B(A)$, such that the summations of spin configurations in $A_{1}$ and $B_{1}$ are interchangeable and can be factorized out explicitly, as shown in Eq. (11). 
where $M_{1}=\frac{1}{4}\left|\psi_{1}\right\rangle\left\langle\psi_{1}\right|$ and $M_{2}=\frac{1}{4}\left|\psi_{2}\right\rangle\left\langle\psi_{2}\right|$ are four-byfour matrices with $\left|\psi_{1}\right\rangle=\frac{1}{2}(|0\rangle+|1\rangle) \otimes(|0\rangle-|1\rangle)$ and $\left|\psi_{2}\right\rangle=\frac{1}{2}(|0\rangle-|1\rangle) \otimes(|0\rangle-|1\rangle)$, respectively; 0 is a zero matrix of dimension $2^{N_{A_{1}}} \times 2^{N_{A_{1}}}$, with $N_{A_{1}}$ denoting the number of spins in subregion $A_{1}$. From Eq. (12), the eigenvalues of $\rho_{A}$ can readily be obtained. Note that $\rho_{A}$ has only four nonzero eigenvalues that are degenerate and equal to $\frac{1}{4}$. As a result, the Rényi entropy is given by

$$
S_{\alpha}^{A}=2 \log 2, \quad \forall \alpha .
$$

For the entanglement spectrum, $H_{\text {ent }}$ has a fourfold degeneracy, with the four smallest eigenvalues equal to $2 \log 2$ and the rest infinite. The fourfold degeneracy is a signature of SPT phases [61,124-126].

We expect that this RBM approach would carry over to calculating the entanglement entropy and spectrum for the 2D and 3D toric code states, whose RBM representation has already been given in Ref. [51], although the calculation will be more technically involved. Undoubtedly, like all analytical methods in calculating entanglement, our RBM approach has obvious limitations and cannot be applied, in general, to an arbitrary short-range RBM state. First, given a specific quantum many-body system, there is so far no systematic way to write down its wave function in terms of RBM. Second, we need certain symmetries (such as the translational symmetry) to substantially simplify the equations. Thus, this approach works only in certain specific circumstances. However, we emphasize that our method does not contain sophisticated mathematics and is a completely new approach that has never been considered in the literature.

\section{CONCLUSION AND OUTLOOK}

In summary, we have studied the entanglement properties of neural-network quantum states in the RBM architecture. In particular, we have proved that all short-range RBM states satisfy an area law of entanglement for arbitrary dimensions and bipartition geometry. This result not only immediately implies an area law for the entanglement of the 1D SPT cluster states and the 2D/3D toric code states (with or without anyonic excitations), but it also sheds light on the open problem of proving the entanglement area law for the ground states of local gapped Hamiltonians in higher dimensions. For generic long-range RBM states with random parameters, we numerically studied their entanglement entropy and spectrum. We found that (i) the averaged entanglement entropy follows a volume law but is significantly smaller than the Page entropy for random pure states and that (ii) their entanglement spectrum has no universal part associated with random matrix theory and manifests a Poisson-type level statistics. In addition, we analytically constructed families of RBM states (in both 1D and 2D) with maximal volumelaw entanglement, which cannot be represented efficiently in terms of matrix product states or tensor-network states.
For these states, the RBM representation is remarkably efficient, requiring only a small number of parameters scaling linearly with the system size. These results explicitly show, in an exact fashion, the remarkable power of artificial neural networks in describing quantum states with massive entanglement. Unlike MPS or tensor-network states, entanglement is not the limiting factor for the efficiency of the neural-network representation of quantum many-body states. Through reinforcement learning of a modified Haldane-Shastry model, we have shown that RBM is capable of calculating the ground state, which has power-law entanglement. The corresponding groundstate energy and correlations can also be efficiently obtained. Finally, we also demonstrated, through a concrete example, that the RBM representation could be used as a tool to analytically compute the entanglement entropy and spectrum for finite systems. Our results reveal some crucial aspects of the data structures of neural-network quantum states and provide a useful guide for the practical applications of machine-learning techniques in solving quantum many-body problems.

Many open questions remain. First, what are the limiting factors for RBMs in efficiently representing quantum states? In the future, it would be interesting and important to find the necessary and sufficient conditions under which a many-body state can be represented efficiently by neural networks, as well as to discover how to convert a general quantum state satisfying these conditions into a RBM. These findings would help develop new machine-learning algorithms for solving many-body problems and advance the understanding, from a physical perspective [127], of the power of machine learning itself. It would also be interesting to study entanglement properties in other types of artificial-neural-network states $[33,34]$. Another interesting direction worth more investigation is the relation between the MPS or tensor-network representation and the neuralnetwork representation. In this context, we note a recent work on supervised machine learning with quantuminspired tensor networks, where the tensor network representation has been shown useful in training neural networks in a supervised way [128]. From Sec. III, we now know that all short-range RBM states in $1 \mathrm{D}$ can be represented in terms of MPS. What about higher dimensions and the inverse statement? Can we rewrite all states bearing a MPS or tensor-network representation with small bond dimensions in terms of short-range RBMs? These questions are worth exploring in the future.

\section{ACKNOWLEDGMENTS}

We thank Frank Verstraete, Roger Melko, Lei Wang, Giuseppe Carleo, Zlatko Papic, Ignacio Cirac, Mikhail D. Lukin, Subir Sachdev, and Matthias Troyer for helpful discussions. D. L. D. thanks Zhengyu Zhang for his help in developing the code for the reinforcement learning calculations. This work is supported by JQI-NSF-PFC and 
LPS-MPO-CMTC. X.L. acknowledges support by the Start-Up Fund of Fudan University. D. L. D. thanks the hospitality of the Kavli Institute for Theoretical Physics (KITP), where this work was partially done. We acknowledge the University of Maryland for the supercomputing resources that were made available in conducting the research reported in this paper.

[1] F. Verstraete, Quantum Hamiltonian Complexity: Worth the Wait, Nat. Phys. 11, 524 (2015).

[2] S. Gharibian, Y. Huang, Z. Landau, and S. W. Shin, Quantum Hamiltonian Complexity, Found. Trends Theor. Comput. Sci. 10, 159 (2015).

[3] T. J. Osborne, Hamiltonian Complexity, Rep. Prog. Phys. 75, 022001 (2012).

[4] J. Eisert, M. Cramer, and M. B. Plenio, Colloquium: Area Laws for the Entanglement Entropy, Rev. Mod. Phys. 82, 277 (2010).

[5] M. B. Hastings, An Area Law for One-Dimensional Quantum Systems, J. Stat. Mech. (2007) P08024.

[6] M. Friesdorf, A. H. Werner, W. Brown, V. B. Scholz, and J. Eisert, Many-Body Localization Implies that Eigenvectors Are Matrix-Product States, Phys. Rev. Lett. 114, 170505 (2015).

[7] M. Fannes, B. Nachtergaele, and R. F. Werner, Finitely Correlated States on Quantum Spin Chains, Commun. Math. Phys. 144, 443 (1992).

[8] D. Perez-Garcia, F. Verstraete, M. Wolf, and J. Cirac, Matrix Product State Representations, Quantum Inf. Comput. 7, 401 (2007).

[9] U. Schollwöck, The Density-Matrix Renormalization Group in the Age of Matrix Product States, Ann. Phys. (Amsterdam) 326, 96 (2011).

[10] F. Verstraete, V. Murg, and J. I. Cirac, Matrix Product States, Projected Entangled Pair States, and Variational Renormalization Group Methods for Quantum Spin Systems, Adv. Phys. 57, 143 (2008).

[11] Z.-C. Gu and X.-G. Wen, Tensor-Entanglement-Filtering Renormalization Approach and Symmetry-Protected Topological Order, Phys. Rev. B 80, 155131 (2009).

[12] R. Orús, A Practical Introduction to Tensor Networks: Matrix Product States and Projected Entangled Pair States, Ann. Phys. (Amsterdam) 349, 117 (2014).

[13] X. Chen, Z.-C. Gu, Z.-X. Liu, and X.-G. Wen, SymmetryProtected Topological Orders in Interacting Bosonic Systems, Science 338, 1604 (2012).

[14] X. Chen, Z.-C. Gu, Z.-X. Liu, and X.-G. Wen, Symmetry Protected Topological Orders and the Group Cohomology of Their Symmetry Group, Phys. Rev. B 87, 155114 (2013).

[15] B. Swingle, Entanglement Renormalization and Holography, Phys. Rev. D 86, 065007 (2012).

[16] B. Swingle, Constructing Holographic Spacetimes Using Entanglement Renormalization, [Phys. Rev. D (to be published)].

[17] S. R. White, Density Matrix Formulation for Quantum Renormalization Groups, Phys. Rev. Lett. 69, 2863 (1992).
[18] U. Schollwöck, The Density-Matrix Renormalization Group, Rev. Mod. Phys. 77, 259 (2005).

[19] G. Vidal, Efficient Classical Simulation of Slightly Entangled Quantum Computations, Phys. Rev. Lett. 91, 147902 (2003).

[20] G. Vidal, Classical Simulation of Infinite-Size Quantum Lattice Systems in One Spatial Dimension, Phys. Rev. Lett. 98, 070201 (2007).

[21] G. Vidal, Class of Quantum Many-Body States that Can Be Efficiently Simulated, Phys. Rev. Lett. 101, 110501 (2008).

[22] G. Carleo and M. Troyer, Solving the Quantum Many-Body Problem with Artificial Neural Networks, Science 355, 602 (2017).

[23] R. S. Michalski, J. G. Carbonell, and T. M. Mitchell, Machine Learning: An Artificial Intelligence Approach (Springer Science \& Business Media, Berlin, 2013).

[24] M. Jordan and T. Mitchell, Machine Learning: Trends, Perspectives, and Prospects, Science 349, 255 (2015).

[25] Y. LeCun, Y. Bengio, and G. Hinton, Deep Learning, Nature 521, 436 (2015).

[26] R. Biswas et al., Application of Machine Learning Algorithms to the Study of Noise Artifacts in GravitationalWave Data, Phys. Rev. D 88, 062003 (2013).

[27] B. P. Abbott et al. (LIGO Scientific Collaboration and Virgo Collaboration), Observation of Gravitational Waves from a Binary Black Hole Merger, Phys. Rev. Lett. 116, 061102 (2016).

[28] M. Pasquato, Detecting Intermediate Mass Black Holes in Globular Clusters with Machine Learning, arXiv:1606.08548.

[29] S. V. Kalinin, B. G. Sumpter, and R. K. Archibald, Big-Deep-Smart Data in Imaging for Guiding Materials Design, Nat. Mater. 14, 973 (2015).

[30] S. S. Schoenholz, E. D. Cubuk, D. M. Sussman, E. Kaxiras, and A. J. Liu, A Structural Approach to Relaxation in Glassy Liquids, Nat. Phys. 12, 469 (2016).

[31] L.-F. Arsenault, O. A. von Lilienfeld, and A. J. Millis, Machine Learning for Many-Body Physics: Efficient Solution of Dynamical Mean-Field Theory, arXiv:1506.08858.

[32] Y. Zhang and E.-A. Kim, Triangular Quantum Loop Topography for Machine Learning, arXiv:1611.01518 [Phys. Rev. Lett. (to be published)]

[33] J. Carrasquilla and R. G. Melko, Machine Learning Phases of Matter, Nat. Phys. online publication (2017).

[34] E. P. van Nieuwenburg, Y.-H. Liu, and S. D. Huber, Learning Phase Transitions by Confusion, Nat. Phys. advance online publication (2017).

[35] L. Wang, Discovering Phase Transitions with Unsupervised Learning, Phys. Rev. B 94, 195105 (2016).

[36] G. Torlai and R. G. Melko, Learning Thermodynamics with Boltzmann Machines, Phys. Rev. B 94, 165134 (2016).

[37] P. Broecker, J. Carrasquilla, R. G. Melko, and S. Trebst, Machine Learning Quantum Phases of Matter Beyond the Fermion Sign Problem, arXiv:1608.07848 [Phys. Rev. X (to be published)].

[38] K. Ch'ng, J. Carrasquilla, R. G. Melko, and E. Khatami, Machine Learning Phases of Strongly Correlated Fermions, arXiv:1609.02552 [Phys. Rev. Lett. (to be published)]. 
[39] G. Torlai and R. G. Melko, A Neural Decoder for Topological Codes, arXiv:1610.04238 [Phys. Rev. Lett. (to be published)].

[40] M. H. Amin, E. Andriyash, J. Rolfe, B. Kulchytskyy, and R. Melko, Quantum Boltzmann Machine, arXiv:1601.02036.

[41] J. Liu, Y. Qi, Z. Y. Meng, and L. Fu, Self-Learning Monte Carlo Method, Phys. Rev. B 95, 041101 (2017).

[42] L. Huang and L. Wang, Accelerate Monte Carlo Simulations with Restricted Boltzmann Machines, Phys. Rev. B 95, 035105 (2017).

[43] K.-I. Aoki and T. Kobayashi, Restricted Boltzmann Machines for the Long Range Ising Models, Mod. Phys. Lett. B 30, 1650401 (2016).

[44] J. Biamonte, P. Wittek, N. Pancotti, P. Rebentrost, N. Wiebe, and S. Lloyd, Quantum Machine Learning, arXiv:1611.09347.

[45] C. Bény, Deep Learning, and the Renormalization Group, arXiv:1301.3124.

[46] P. Mehta and D. J. Schwab, An Exact Mapping Between the Variational Renormalization Group and Deep Learning, arXiv:1410.3831.

[47] N. M. Tubman, Measuring Quantum Entanglement, Machine Learning and Wave Function Tomography: Bridging Theory and Experiment with the Quantum Gas Microscope, arXiv:1609.08142.

[48] G. E. Hinton and R. R. Salakhutdinov, Reducing the Dimensionality of Data with Neural Networks, Science 313, 504 (2006).

[49] R. Salakhutdinov, A. Mnih, and G. Hinton, Restricted Boltzmann Machines for Collaborative Filtering, in Proceedings of the 24th International Conference on Machine Learning (ACM, New York, 2007), pp. 791-798.

[50] H. Larochelle and Y. Bengio, Classification Using Discriminative Restricted Boltzmann Machines, in Proceedings of the 25th International Conference on Machine Learning (ACM, New York, 2008), pp. 536-543.

[51] D.-L. Deng, X. Li, and S. D. Sarma, Exact Machine Learning Topological States, arXiv:1609.09060.

[52] C. Chamon, A. Hamma, and E. R. Mucciolo, Emergent Irreversibility and Entanglement Spectrum Statistics, Phys. Rev. Lett. 112, 240501 (2014).

[53] S. Sorella, M. Casula, and D. Rocca, Weak Binding Between Two Aromatic Rings: Feeling the van der Waals Attraction by Quantum Monte Carlo Methods, J. Chem. Phys. 127, 014105 (2007).

[54] F. D. M. Haldane, Exact Jastrow-Gutzwiller ResonatingValence-Bond Ground State of the Spin-(1/2 Antiferromagnetic Heisenberg Chain with $1 / \mathrm{r}^{2}$ Exchange, Phys. Rev. Lett. 60, 635 (1988).

[55] B. S. Shastry, Exact Solution of an $S=1 / 2$ Heisenberg Antiferromagnetic Chain with Long-Ranged Interactions, Phys. Rev. Lett. 60, 639 (1988).

[56] A. Coates, A. Y. Ng, and H. Lee, An Analysis of SingleLayer Networks in Unsupervised Feature Learning, in International Conference on Artificial Intelligence and Statistics, 2011, pp. 215-223.

[57] A. N. Kolmogorov, On the Representation of Continuous Functions of Many Variables by Superposition of Continuous Functions of One Variable and Addition, Transl.Am. Math. Soc. 28, 55 (1963).
[58] N. Le Roux and Y. Bengio, Representational Power of Restricted Boltzmann Machines and Deep Belief Networks, Neural Comput. 20, 1631 (2008).

[59] K. Hornik, Approximation Capabilities of Multilayer Feedforward Networks, Neural Netw. 4, 251 (1991).

[60] L. Amico, R. Fazio, A. Osterloh, and V. Vedral, Entanglement in Many-Body Systems, Rev. Mod. Phys. 80, 517 (2008).

[61] H. Li and F. D. M. Haldane, Entanglement Spectrum as a Generalization of Entanglement Entropy: Identification of Topological Order in Non-Abelian Fractional Quantum Hall Effect States, Phys. Rev. Lett. 101, 010504 (2008).

[62] M. Levin and X.-G. Wen, Detecting Topological Order in a Ground State Wave Function, Phys. Rev. Lett. 96, 110405 (2006).

[63] A. Kitaev and J. Preskill, Topological Entanglement Entropy, Phys. Rev. Lett. 96, 110404 (2006).

[64] J. Biddle, M. R. Peterson, and S. Das Sarma, Entanglement Measures for Quasi-Two-Dimensional Fractional Quantum Hall States, Phys. Rev. B 84, 125141 (2011).

[65] A. Osterloh, L. Amico, G. Falci, and R. Fazio, Scaling of Entanglement Close to a Quantum Phase Transition, Nature 416, 608 (2002).

[66] T. J. Osborne and M. A. Nielsen, Entanglement in a Simple Quantum Phase Transition, Phys. Rev. A 66, 032110 (2002).

[67] S.-J. Gu, S.-S. Deng, Y.-Q. Li, and H.-Q. Lin, Entanglement and Quantum Phase Transition in the Extended Hubbard Model, Phys. Rev. Lett. 93, 086402 (2004).

[68] A. Pal and D. A. Huse, Many-Body Localization Phase Transition, Phys. Rev. B 82, 174411 (2010).

[69] B. Bauer and C. Nayak, Area Laws in a Many-Body Localized State and Its Implications for Topological Order, J. Stat. Mech. Theor. Exp. P09005 (2013).

[70] R. Nandkishore and D. A. Huse, Many-Body Localization and Thermalization in Quantum Statistical Mechanics, Annu. Rev. Condens. Matter Phys. 6, 15 (2015).

[71] C. M. Alves and D. Jaksch, Multipartite Entanglement Detection in Bosons, Phys. Rev. Lett. 93, 110501 (2004).

[72] A. J. Daley, H. Pichler, J. Schachenmayer, and P. Zoller, Measuring Entanglement Growth in Quench Dynamics of Bosons in an Optical Lattice, Phys. Rev. Lett. 109, 020505 (2012).

[73] D. A. Abanin and E. Demler, Measuring Entanglement Entropy of a Generic Many-Body System with a Quantum Switch, Phys. Rev. Lett. 109, 020504 (2012).

[74] P. Hauke, M. Heyl, L. Tagliacozzo, and P. Zoller, Measuring Multipartite Entanglement through Dynamic Susceptibilities, Nat. Phys. (2016).

[75] H. Pichler, G. Zhu, A. Seif, P. Zoller, and M. Hafezi, Measurement Protocol for the Entanglement Spectrum of Cold Atoms, Phys. Rev. X 6, 041033 (2016).

[76] R. Islam, R. Ma, P. M. Preiss, M. E. Tai, A. Lukin, M. Rispoli, and M. Greiner, Measuring Entanglement Entropy in a Quantum Many-Body System, Nature 528, 77 (2015).

[77] A. M. Kaufman, M.E. Tai, A. Lukin, M. Rispoli, R. Schittko, P. M. Preiss, and M. Greiner, Quantum Thermalization through Entanglement in an Isolated Many-Body System, Science 353, 794 (2016). 
[78] S. Hawking, J. Maldacena, and A. Strominger, DeSitter Entropy, Quantum Entanglement and AdS/CFT, J. High Energy Phys. 05 (2001) 001.

[79] J. D. Bekenstein, Black Holes and Entropy, Phys. Rev. D 7, 2333 (1973).

[80] L. Bombelli, R. K. Koul, J. Lee, and R. D. Sorkin, Quantum Source of Entropy for Black Holes, Phys. Rev. D 34, 373 (1986).

[81] M. Srednicki, Entropy and Area, Phys. Rev. Lett. 71, 666 (1993).

[82] E. H. Lieb and D. W. Robinson, The Finite Group Velocity of Quantum Spin Systems, Commun. Math. Phys. 28, 251 (1972).

[83] I. Arad, A. Kitaev, Z. Landau, and U. Vazirani, An Area Law and Sub-exponential Algorithm for $1 D$ Systems, arXiv:1301.1162.

[84] Y. Huang, Area Law in One Dimension: Degenerate Ground States and Renyi Entanglement Entropy, arXiv:1403.0327 [Phys. Rev. B (to be published)].

[85] F. G. Brandão and M. Horodecki, An Area Law for Entanglement from Exponential Decay of Correlations, Nat. Phys. 9, 721 (2013).

[86] F. Verstraete and J. I. Cirac, Matrix Product States Represent Ground States Faithfully, Phys. Rev. B 73, 094423 (2006).

[87] N. Schuch, M. M. Wolf, F. Verstraete, and J. I. Cirac, Entropy Scaling and Simulability by Matrix Product States, Phys. Rev. Lett. 100, 030504 (2008).

[88] V. Khemani, F. Pollmann, and S. L. Sondhi, Obtaining Highly Excited Eigenstates of Many-Body Localized Hamiltonians by the Density Matrix Renormalization Group Approach, Phys. Rev. Lett. 116, 247204 (2016).

[89] D. N. Page, Average Entropy of a Subsystem, Phys. Rev. Lett. 71, 1291 (1993).

[90] V. Khemani, S. Lim, D. Sheng, and D. A. Huse, Critical Properties of the Many-Body Localization Transition, arXiv:1607.05756 [Phys. Rev. X (to be published)].

[91] C. Mejía-Monasterio, G. Benenti, G. G. Carlo, and G. Casati, Entanglement Across a Transition to Quantum Chaos, Phys. Rev. A 71, 062324 (2005).

[92] D. Harlow, Jerusalem Lectures on Black Holes and Quantum Information, Rev. Mod. Phys. 88, 015002 (2016).

[93] D. N. Page, Information in Black Hole Radiation, Phys. Rev. Lett. 71, 3743 (1993).

[94] J. A. Kjäll, J. H. Bardarson, and F. Pollmann, Many-Body Localization in a Disordered Quantum Ising Chain, Phys. Rev. Lett. 113, 107204 (2014).

[95] X. Li, D.-L. Deng, Y.-L. Wu, and S. D. Sarma, Statistical Bubble Localization with Random Interactions, Phys. Rev. B 95, 020201 (2017).

[96] L. Susskind, Entanglement Is Not Enough, Fort. Phys. 64, 49 (2016).

[97] Z.-C. Yang, C. Chamon, A. Hamma, and E. R. Mucciolo, Two-Component Structure in the Entanglement Spectrum of Highly Excited States, Phys. Rev. Lett. 115, 267206 (2015).

[98] S. D. Geraedts, R. Nandkishore, and N. Regnault, ManyBody Localization and Thermalization: Insights from the Entanglement Spectrum, Phys. Rev. B 93, 174202 (2016).

[99] J. I. Cirac, D. Poilblanc, N. Schuch, and F. Verstraete, Entanglement Spectrum and Boundary Theories with
Projected Entangled-Pair States, Phys. Rev. B 83, 245134 (2011).

[100] P. Calabrese and A. Lefevre, Entanglement Spectrum in One-Dimensional Systems, Phys. Rev. A 78, 032329 (2008).

[101] J. R. Garrison and T. Grover, Does a Single Eigenstate Encode the Full Hamiltonian?, arXiv:1503.00729 [Phys. Rev. X (to be published)].

[102] V. A. Marčenko and L. A. Pastur, Distribution of Eigenvalues for Some Sets of Random Matrices, Math. USSR-Sbornik 1, 457 (1967).

[103] T. A. Brody, J. Flores, J. B. French, P. A. Mello, A. Pandey, and S.S. M. Wong, Random-Matrix Physics: Spectrum and Strength Fluctuations, Rev. Mod. Phys. 53, 385 (1981).

[104] M. V. Berry and M. Tabor, Level Clustering in the Regular Spectrum, Proc. R. Soc. A 356, 375 (1977).

[105] O. Bohigas, M. J. Giannoni, and C. Schmit, Characterization of Chaotic Quantum Spectra and Universality of Level Fluctuation Laws, Phys. Rev. Lett. 52, 1 (1984).

[106] G. Akemann, J. Baik, and P. Di Francesco, The Oxford Handbook of Random Matrix Theory (Oxford University Press, New York, 2011).

[107] Y. Y. Atas, E. Bogomolny, O. Giraud, and G. Roux, Distribution of the Ratio of Consecutive Level Spacings in Random Matrix Ensembles, Phys. Rev. Lett. 110, 084101 (2013).

[108] J. Haegeman, C. Lubich, I. Oseledets, B. Vandereycken, and F. Verstraete, Unifying Time Evolution and Optimization with Matrix Product States, Phys. Rev. B 94, 165116 (2016).

[109] T. Graß and M. Lewenstein, Trapped-Ion Quantum Simulation of Tunable-Range Heisenberg Chains, EPJ Quantum Techno. 1, 1 (2014).

[110] Z. Gong, Ph.D. thesis, University of Michigan (2013).

[111] B.-Q. Jin and V. E. Korepin, Quantum Spin Chain, Toeplitz Determinants and the Fisher Hartwig Conjecture, J. Stat. Phys. 116, 79 (2004).

[112] A. R. Its, B.-Q. Jin, and V. E. Korepin, Entanglement in the XY Spin Chain, J. Phys. A 38, 2975 (2005).

[113] J. Eisert and M. Cramer, Single-Copy Entanglement in Critical Quantum Spin Chains, Phys. Rev. A 72, 042112 (2005).

[114] J. P. Keating and F. Mezzadri, Entanglement in Quantum Spin Chains, Symmetry Classes of Random Matrices, and Conformal Field Theory, Phys. Rev. Lett. 94, 050501 (2005).

[115] C. Holzhey, F. Larsen, and F. Wilczek, Geometric and Renormalized Entropy in Conformal Field Theory, Nucl. Phys. B424, 443 (1994).

[116] P. Calabrese and J. Cardy, Entanglement Entropy and Quantum Field Theory, J. Stat. Mech. (2004) P06002.

[117] C. Callan and F. Wilczek, On Geometric Entropy, Phys. Lett. B 333, 55 (1994).

[118] A. Hamma, R. Ionicioiu, and P. Zanardi, Bipartite Entanglement and Entropic Boundary Law in Lattice Spin Systems, Phys. Rev. A 71, 022315 (2005).

[119] A. Hamma, R. Ionicioiu, and P. Zanardi, Ground State Entanglement and Geometric Entropy in the Kitaev Model, Phys. Lett. A 337, 22 (2005). 
[120] W. Son, L. Amico, and V. Vedral, Topological Order in 1D Cluster State Protected by Symmetry, Quantum Inf. Process. 11, 1961 (2012).

[121] M. Hein, J. Eisert, and H. J. Briegel, Multiparty Entanglement in Graph States, Phys. Rev. A 69, 062311 (2004).

[122] R. Raussendorf, D. E. Browne, and H. J. Briegel, Measurement-Based Quantum Computation on Cluster States, Phys. Rev. A 68, 022312 (2003).

[123] M. A. Nielsen, Cluster-State Quantum Computation, Rep. Math. Phys. 57, 147 (2006).

[124] A. Chandran, M. Hermanns, N. Regnault, and B. A. Bernevig, Bulk-Edge Correspondence in Entanglement Spectra, Phys. Rev. B 84, 205136 (2011).
[125] L. Fidkowski, Entanglement Spectrum of Topological Insulators and Superconductors, Phys. Rev. Lett. 104, 130502 (2010).

[126] X.-L. Qi, H. Katsura, and A. W. W. Ludwig, General Relationship Between the Entanglement Spectrum and the Edge State Spectrum of Topological Quantum States, Phys. Rev. Lett. 108, 196402 (2012).

[127] H. W. Lin and M. Tegmark, Why Does Deep and Cheap Learning Work So Well?, arXiv:1608.08225 [Phys. Rev. X (to be published)].

[128] E. M. Stoudenmire and D. J. Schwab, Supervised Learning with Quantum-Inspired Tensor Networks, Adv. Neural Inf. Process. Syst. 29, 4799 (2016). 